\title{
Reader-Writer Synchronization for Shared-Memory Multiprocessor Real-Time Systems*
}

\author{
Björn B. Brandenburg and James H. Anderson \\ Department of Computer Science, University of North Carolina at Chapel Hill
}

\begin{abstract}
Reader preference, writer preference, and task-fair readerwriter locks are shown to cause undue blocking in multiprocessor real-time systems. A new phase-fair reader-writer lock is proposed as an alternative that significantly reduces worstcase blocking for readers and an efficient local-spin implementation is provided. Both task-and phase-fair locks are evaluated and contrasted to mutex locks in terms of hard and soft real-time schedulability under consideration of runtime overheads on a multicore computer.
\end{abstract}

\section{Introduction}

With the transition to multicore architectures by most (if not all) major chip manufacturers, multiprocessors are now a standard deployment platform for (soft) real-time applications. This has led to renewed interest in real-time multiprocessor scheduling and synchronization algorithms (see [11, 14, 15, 17] for recent comparative studies and relevant references). However, prior work on synchronization has been somewhat limited, being mostly focused on mechanisms that ensure strict mutual exclusion (mutex). Reader-writer (RW) synchronization, which requires mutual exclusion only for updates (and not for reads) has not been considered in prior work on real-time shared-memory multiprocessor systems despite its great practical relevance.

The need for RW synchronization arises naturally in many situations. Two common examples are few-producers/manyconsumers relationships (e.g., obtaining and distributing sensor data) and rarely-changing shared state (e.g., configuration data). As an example for the former, consider a robot such as TU Berlin's autonomous helicopter Marvin [29]: its GPS receiver updates the current position estimate 20 times per second, and the latest position estimate is read at various rates by a flight controller and by image acquisition, camera targeting, and communication modules. An example of rarely-changing shared data occurs in work of Gore et al. [21], who employed RW locks to optimize latency in a real-time notification service. In their system, every incoming event must be matched against a shared subscription lookup table to determine the set of subscribing clients. Since events occur very frequently and changes to the table occur only very rarely, the use of a regu-

*Work supported by IBM, Intel, and Sun Corps.; NSF grants CNS 0834270, CNS 0834132, and CNS 0615197; and ARO grant W911NF-06$1-0425$. lar mutex lock "can unnecessarily reduce [concurrency] in the critical path of event propagation." [21]

In practice, RW locks are in wide-spread use since they are supported by all POSIX-compliant real-time operating systems. However, to the best of our knowledge, such locks have not been analyzed in the context of multiprocessor real-time systems from a schedulability perspective. In fact, RW locks that are subject to starvation have been suggested to practitioners without much concern for schedulability [24]. This highlights the need for a well-understood analytically-sound real-time RW synchronization protocol.

Related work. In work on non-real-time systems, Courtois et al. were the first to investigate RW synchronization and proposed two semaphore-based RW locks [18]: a writer preference lock, wherein writers have higher priority than readers, and a reader preference lock, wherein readers have higher priority than writers. Both are problematic for real-time systems as they can give rise to extended delays and even starvation. To improve reader throughput at the expense of writer throughput in large parallel systems, Hsieh and Weihl proposed a semaphore-based RW lock wherein the lock state is distributed across processors [22]. In work on scalable synchronization on shared-memory multiprocessors, MellorCrummey and Scott proposed spin-based reader preference, writer preference, and task-fair RW locks [28]. In a taskfair RW lock, readers and writers gain access in strict FIFO order, which avoids starvation. In the same work, MellorCrummey and Scott also proposed local-spin versions of their RW locks, in which excessive memory bus traffic under high contention is avoided. An alternative local-spin implementation of task-fair RW locks was later proposed by Krieger et al. [23]. A probabilistic performance analysis of task-fair RW locks wherein reader arrivals are modeled as a Poisson process was conducted by Reiman and Wright [31].

In work on uniprocessor real-time systems, two relevant suspension-based protocols have been proposed: Baker's stack resource policy [3] and Rajkumar's read-write priority ceiling protocol [30]. The latter has also been studied in the context of distributed real-time databases [30].

An alternative to locking is the use of specialized nonblocking algorithms [1]. However, compared to lock-based RW synchronization, which allows in-place updates, nonblocking approaches usually require additional memory, incur significant copying or retry overheads, and are less general. ${ }^{1}$

\footnotetext{
${ }^{1}$ In non-blocking read-write algorithms, the value to be written must be
} 
In this paper, we focus on lock-based RW synchronization in cache-coherent shared-memory multiprocessor systems.

Contributions. The contributions of this paper are as follows: (i) We study the applicability of existing RW locks to real-time systems and derive worst-case blocking bounds; (ii) we propose a novel type of RW lock based on the concept of "phase-fairness," derive corresponding worst-case blocking bounds, and present two efficient implementations; (iii) we report on the results of an extensive performance evaluation of RW synchronization choices in terms of hard and soft real-time schedulability under consideration of system overheads. These experiments show that employing "phase-fair" RW locks can significantly improve real-time performance.

The rest of this paper is organized as follows. We summarize relevant background in Sec. 2, and discuss our synchronization protocol and other RW lock choices in Sec. 3. An empirical performance evaluation is presented in Sec. 4, followed by our conclusions in Sec. 5 .

\section{Background}

We consider the scheduling of a system of sporadic tasks, denoted $T_{1}, \ldots, T_{N}$, on $m$ processors. The $j^{\text {th }}$ job (or invocation) of task $T_{i}$ is denoted $T_{i}^{j}$. Such a job $T_{i}^{j}$ becomes available for execution at its release time, $\mathrm{r}\left(T_{i}^{j}\right)$. Each task $T_{i}$ is specified by its worst-case (per-job) execution cost, $\mathrm{e}\left(T_{i}\right)$, its period, $\mathrm{p}\left(T_{i}\right)$, and its relative deadline, $\mathrm{d}\left(T_{i}\right) \geq \mathrm{e}\left(T_{i}\right)$. A job $T_{i}^{j}$ should complete execution by its absolute deadline, $\mathrm{r}\left(T_{i}^{j}\right)+\mathrm{d}\left(T_{i}\right)$, and is tardy if it completes later. The spacing between job releases must satisfy $\mathrm{r}\left(T_{i}^{j+1}\right) \geq \mathrm{r}\left(T_{i}^{j}\right)+\mathrm{p}\left(T_{i}\right)$. A job that has not completed execution is either preemptable or non-preemptable. A job scheduled on a processor can only be preempted when it is preemptable. Task $T_{i}$ 's utilization (or weight) reflects the processor share that it requires and is given by $\mathrm{e}\left(T_{i}\right) / \mathrm{p}\left(T_{i}\right)$.

Multiprocessor scheduling. There are two fundamental approaches to scheduling sporadic tasks on multiprocessors global and partitioned. With global scheduling, processors are scheduled by selecting jobs from a single, shared queue, whereas with partitioned scheduling, each processor has a private queue and is scheduled independently using a uniprocessor scheduling policy (hybrid approaches exist, too [16]). Tasks are statically assigned to processors under partitioning. As a consequence, under partitioned scheduling, all jobs of a task execute on the same processor, whereas migrations may occur in globally-scheduled systems. A discussion of the tradeoffs between global and partitioned scheduling is beyond the scope of this paper and the interested reader is referred to prior studies $[14,17]$.

We consider one representative algorithm from each category: in the partitioned case, the partitioned EDF (P-EDF)

pre-determined and cannot depend on the current state of the shared object. With locks, an update can be computed based on the current value. algorithm, wherein the earliest-deadline-first (EDF) algorithm is used on each processor, and in the global case, the global EDF (G-EDF) algorithm. However, the synchronization algorithms considered herein apply equally to other scheduling algorithms that assign each job a fixed priority, including partitioned static-priority (P-SP) scheduling. Further, we consider both hard real-time (HRT) systems in which deadlines should not be missed, and soft real-time systems (SRT) in which bounded deadline tardiness is permissible.

Resources. When a job $T_{i}^{j}$ is going to update (observe) the state of a resource $\ell$, it issues a write (read) request $\mathcal{W}_{\ell}\left(\mathcal{R}_{\ell}\right)$ for $\ell$ and is said to be a writer (reader). In the following discussion, which applies equally to read and write requests, we denote a request for $\ell$ of either kind as $\mathcal{X}_{\ell}$.

$\mathcal{X}_{\ell}$ is satisfied as soon as $T_{i}^{j}$ holds $\ell$, and completes when $T_{i}^{j}$ releases $\ell .\left|\mathcal{X}_{\ell}\right|$ denotes the maximum time that $T_{i}^{j}$ will hold $\ell$. $T_{i}^{j}$ becomes blocked on $\ell$ if $\mathcal{X}_{\ell}$ cannot be satisfied immediately. (A resource can be held by multiple jobs simultaneously only if they are all readers.) If $T_{i}^{j}$ issues another request $\mathcal{X}^{\prime}$ before $\mathcal{X}$ is complete, then $\mathcal{X}^{\prime}$ is nested within $\mathcal{X}$. We assume nesting is proper, i.e., $\mathcal{X}^{\prime}$ must complete no later than $\mathcal{X}$ completes. An outermost request is not nested within any other request. A resource is global if it can be requested concurrently by jobs scheduled on different processors, and local otherwise.

Infrequent requests. Some infrequently read or updated resources may not be requested by every job of a task. We associate a request period $\operatorname{rp}(\mathcal{X}) \in \mathbb{N}$ with every request $\mathcal{X}$ to allow infrequent resource requests to be represented in the task model without introducing unnecessary pessimism. The request period limits the maximum request frequency: if jobs of $T_{i}$ issue $\mathcal{X}$ and $\operatorname{rp}(\mathcal{X})=k$, then at most every $k$ th job of $T_{i}$ issues $\mathcal{X}$.

For example, consider a task $T_{m}$ that reads a sensor $\ell_{s}$ at a rate of ten samples per second via a read request $\mathcal{R}_{s}$ $\left(\mathrm{p}\left(T_{m}\right)=100 \mathrm{~ms}\right)$ and that updates a shared variable $\ell_{a}$ storing a history of average readings once every two seconds via a write request $\mathcal{W}_{a}$. In this case, assuming that every job of $T_{m}$ issues $\mathcal{W}_{a}$ when analyzing contention for $\ell_{a}$ would be unnecessarily pessimistic. To reflect the fact that only every 20th job of $T_{m}$ accesses $\ell_{a}$, we can define $\operatorname{rp}\left(\mathcal{W}_{a}\right)=20$ and $\operatorname{rp}\left(\mathcal{R}_{s}\right)=1$.

When sharing resources in real-time systems, a locking protocol must be employed to both avoid deadlock and bound the maximum duration of blocking. In the following section, we present such a protocol.

\section{Reader-Writer Synchronization}

The flexible multiprocessor locking protocol (FMLP) $[8,12]$ is a real-time mutex protocol based on the principles of flexibility and simplicity that has been shown to compare favorably to previously-proposed protocols $[8,11]$. In this section, 
we present an extended version of the FMLP with support for RW synchronization. We begin by giving a high-level overview of its core design.

The FMLP is considered to be "flexible" for two reasons: it can be used under G-EDF, P-EDF, as well as P-SP scheduling, and it is agnostic regarding whether blocking is via spinning or suspension. Regarding the latter, resources are categorized as either "short" or "long." Short resources are accessed using fair spin locks and long resources are accessed via a semaphore protocol. Whether a resource should be considered short or long is user-defined, but requests for long resources may not be contained within requests for short resources. The terms "short" and "long" arise because (intuitively) spinning is appropriate only for short critical sections, since spinning wastes processor time. However, two recent studies have shown that, in terms of schedulability, spinning is usually preferable to suspending when overheads are considered [11,15]. Based on these trends (and due to space constraints), we restrict our focus to short resources in this paper and delegate RW synchronization of long resources to future work.

\subsection{Reader-Writer Request Rules}

The reader-writer FMLP (RW-FMLP) is realized by the following rules, which are based on those given in [8].

Resource groups. Nesting, which is required to cause a deadlock, tends to occur somewhat infrequently in practice [10]. The FMLP strikes a balance between supporting nesting and optimizing for the common case (no nesting) by organizing resources into resource groups, which are sets of resources that may be requested together. Two resources are in the same group iff there exists a job that requests both resources at the same time. We let $\operatorname{grp}(\ell)$ denote the group that contains $\ell$. Deadlock is avoided by protecting each group by a group lock; before a job can access a resource, it must first acquire its corresponding group lock. ${ }^{2}$

Nesting. Read requests may be freely nested within other read and write requests, but write requests may only be nested within other write requests. We do not permit the nesting of write requests within read requests because this would require an "upgrade" to exclusive access, which is problematic for worst-case blocking analysis.

Requests. If a job $T_{i}^{j}$ issues a read or write request $\mathcal{X}_{\ell}$ for a short resource $\ell$ and $\mathcal{X}_{\ell}$ is outermost, then $T_{i}^{j}$ becomes nonpreemptable and executes the corresponding entry protocol for $\operatorname{grp}(\ell)$ 's group lock (the details of which are discussed in the next section). $\mathcal{X}_{\ell}$ is satisfied once $T_{i}^{j}$ holds $\ell$ 's group lock. When $\mathcal{X}_{\ell}$ completes, $T_{i}^{j}$ releases the group lock and leaves

\footnotetext{
${ }^{2}$ Group-locking is admittedly a very simple deadlock avoidance mechanism; however, the FMLP is the first multiprocessor real-time locking protocol that allows nesting of global resources at all. Obtaining a provably better protocol remains an interesting open question.
}

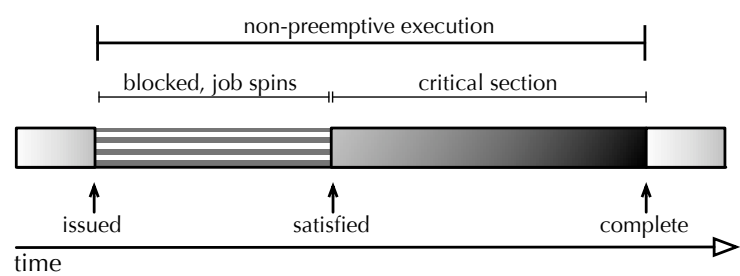

Figure 1: Illustration of an outermost request.

its non-preemptive section. The execution of an outermost request is illustrated in Fig. 1. If $\mathcal{X}_{\ell}$ is not outermost, then it is satisfied immediately: if the outer request is a write request, then $T_{i}^{j}$ already has exclusive access to the group, and if the outer request is a read request, then $\mathcal{X}_{\ell}$ must also be a read request according to the nesting rule. In either case, it is safe to acquire $\ell$.

The RW-FMLP can be integrated with the regular FMLP by replacing the FMLP's short request rules with the aboveprovided rules; regular short FMLP requests are then treated as short write requests.

\subsection{Group Lock Choices}

Group locks are the fundamental unit of locking in the FMLP and thus determine its worst-case blocking behavior. In this section, we consider four group lock choices (one of them a new RW lock) and discuss examples showing how using RW locks and relaxing ordering constraints can significantly reduce worst-case blocking. Bounds on worst-case blocking for all three choices are derived in an extended version of this paper [9].

Task-fair mutex locks are considered here even though they are clearly undesirable for RW synchronization since they are the only spin-based locks for which bounds on worst-case blocking were derived in prior work. Hence, they serve in our experiments (see Sec. 4) as a performance baseline.

Task-fair mutex locks. With task-fair (or FIFO) locks, competing tasks are served strictly in the order that they issue requests. Task-fair mutex locks were employed in previous work on real-time synchronization because they have two desirable properties: first, they can be implemented efficiently [27], and second, they offer strong progress guarantees. Since requests (in the FMLP) are executed nonpreemptively, task-fair locks ensure that a request of a job is blocked once by at most $m-1$ other jobs irrespective of the number of competing requests, which may be desirable as this tends to distribute blocking evenly among tasks.

However, enforcing strict mutual exclusion among readers is unnecessarily restrictive and can in fact cause deadline misses. An example is shown in Fig. 2(a), which depicts jobs of five tasks (two writers, three readers) competing for a resource $\ell$. As $\ell$ 's group lock is a task-fair mutex lock, all requests are satisfied sequentially in the order that they were issued. This unnecessarily delays both $T_{4}$ and $T_{5}$ and causes them to miss their respective deadlines at times 12.5 and 13 . 
Task-fair RW locks. With task-fair RW locks, while requests are still satisfied in strict FIFO order, the mutual exclusion requirement is relaxed so that the lock can be acquired by multiple readers. This can lead to reduced blocking, as shown in Fig. 2(b), which depicts the same arrival sequence as in Fig. 2(a). Note that the read requests of $T_{3}, T_{4}$, and $T_{5}$ are satisfied simultaneously at time 8 , which in turn allows $T_{4}$ and $T_{5}$ to meet their deadlines.

Unfortunately, task-fair RW locks may degrade to mutexlike performance when faced with a pathological request sequence, as is shown in Fig. 2(c). The only difference in task behavior between Fig. 2(b) and Fig. 2(c) is that the arrival times of the jobs of $T_{1}$ and $T_{4}$ have been switched. This causes $\ell$ to be requested first by a reader ( $T_{4}$ at time 2$)$, then by a writer ( $T_{2}$ at time 2.5$)$, then by a reader again $\left(T_{3}\right.$ at time 3$)$, then by another writer ( $T_{1}$ at time 3.5$)$, and finally by the last reader ( $T_{5}$ at time 4$)$. Reader parallelism is eliminated in this scenario and $T_{5}$ misses its deadline at time 13 as a result.

Preference RW locks. In a reader preference lock, readers are statically prioritized over writers, i.e., writers are starved as long as readers issue consecutive requests [18, 28]. The lack of strong progress guarantees for writers makes reader preference locks a problematic choice for real-time systems: one can easily construct examples in which deadlines are missed due to writer starvation. Writer preference locks are an ill choice for similar reasons.

All previously-proposed RW locks fall within one of the categories discussed so far. Thus, the examples discussed above demonstrate that no such lock reliably reduces worstcase blocking (unless there are very few writers).

Phase-fair RW locks. Upon closer inspection, one can identify two root problems of existing RW locks: first, preference locks cause extended blocking due to intervals in which only requests of one kind are satisfied; and second, task-fair RW locks cause extended blocking due to a lack of parallelism when readers are interleaved with writers. A RW lock better suited for real-time systems should avoid these two pitfalls.

These two requirements are captured by the concept of phase-fairness. Phase-fair RW locks have the following properties: (i) reader phases and writer phases alternate; (ii) writers are subject to FIFO ordering with regard to other writers; (iii) all unsatisfied read requests are satisfied at the beginning of a reader phase, ${ }^{3}$ and (iv) if there are incomplete write requests, then read requests are not satisfied until the start of the next reader phase. Properties (i) and (iii) ensure that a read request is never blocked by more than one writer phase and one reader phase irrespective of $m$ and the length of the write request queue. Property (iv) ensures that reader phases end. Properties (i) and (ii) ensure that a write request is never blocked by more than $m-1$ phases. The fact that the bound on read request blocking is $O(1)$ and not $O(m)$ is very significant as multicore platforms become larger.

\footnotetext{
${ }^{3}$ Exactly one write request is satisfied at the beginning of a writer phase.
}
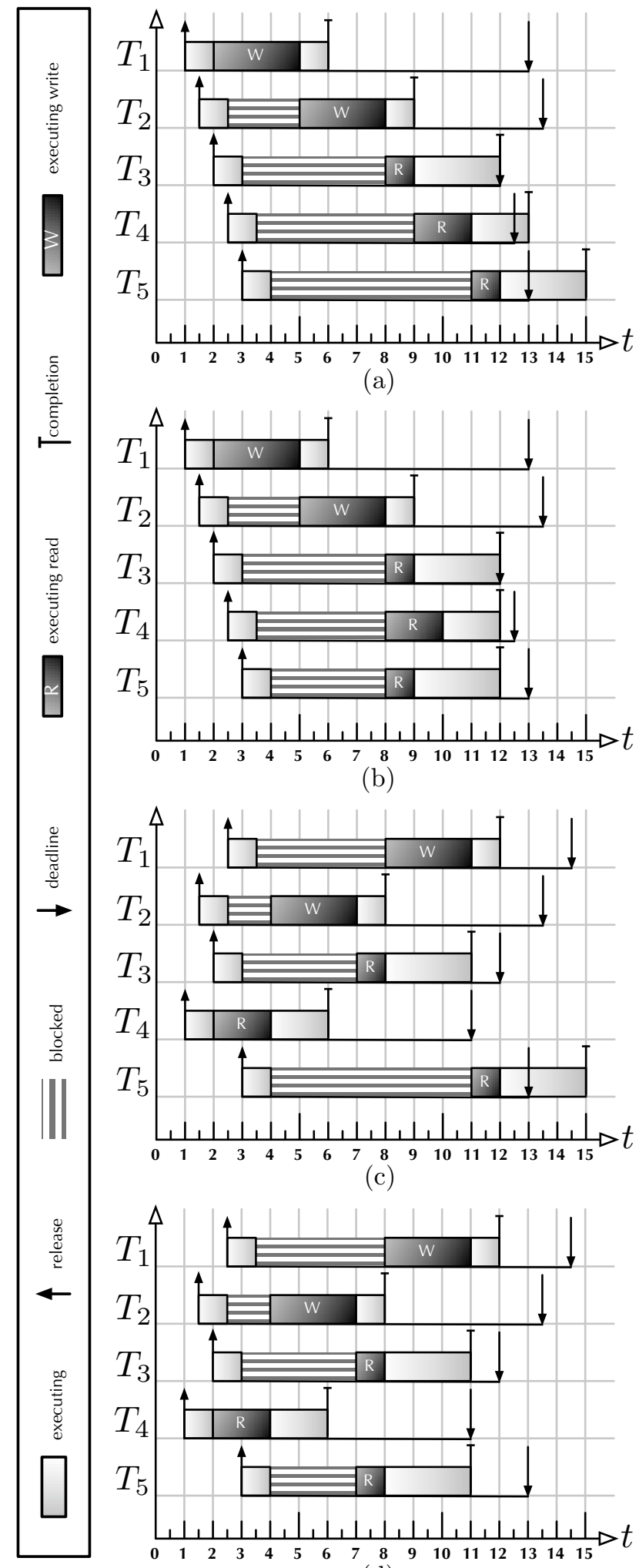

(a)

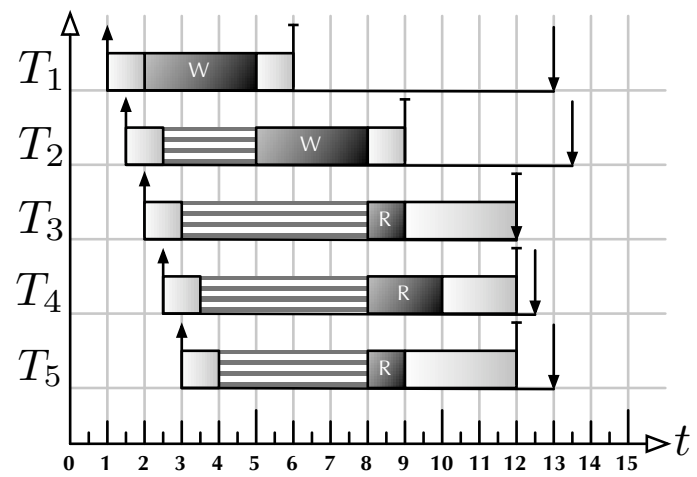

(b)

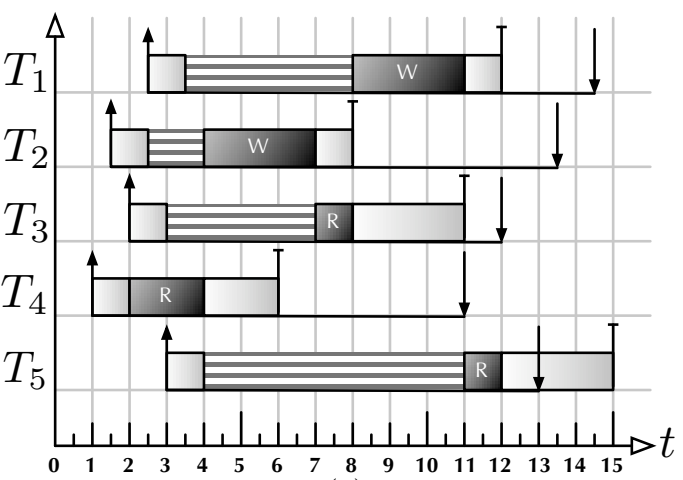

(c)

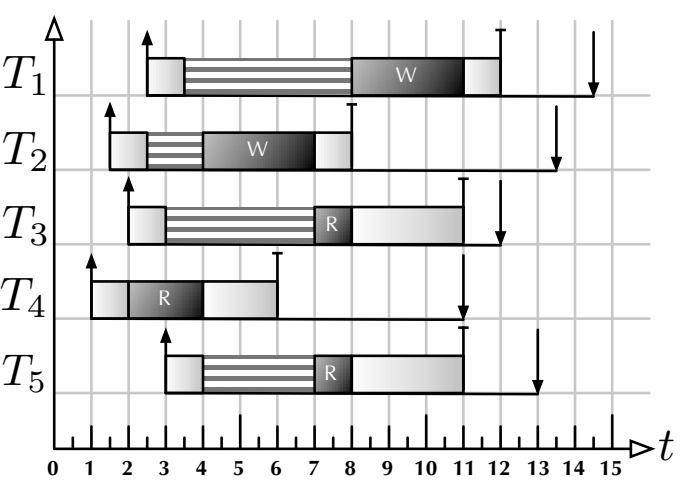

(d)

Figure 2: Example schedules of two writers $\left(T_{1}, T_{2}\right)$ and three readers $\left(T_{3}, T_{4}, T_{5}\right)$ sharing one resource (tasks assigned to different processors). (a) Task-fair mutex group lock. (b) Task-fair RW group lock (best-case arrival sequence). (c) Task-fair RW group lock (worst-case arrival sequence). (d) Phase-fair RW group lock. 
Fig. 2(d) depicts a schedule of the pathological arrival sequence from Fig. 2(c) assuming a phase-fair group lock. $T_{4}$ issues the first read request and thus starts a new reader phase at time 2. $T_{2}$ issues a write request that cannot be satisfied immediately at time 2.5. However, $T_{2}$ 's unsatisfied request prevents the next read request (issued by $T_{3}$ at time 3 ) from being satisfied due to Property (iv). At time 3.5, $T_{1}$ issues a second write request, and at time $4, T_{5}$ issues a final read request. At the same time, $T_{4}$ 's request completes and the first reader phase ends. The first writer phase lasts from time 4 to time 7 when $T_{2}$ 's write request, which was first in the writer FIFO queue, completes. Due to Property (i), this starts the next reader phase, and due to Property (iii), all unsatisfied read requests are satisfied. Note that, when $T_{5}$ 's read request was issued, two write requests were unsatisfied. However, due to the phase-fair bound on read-request blocking, it was only blocked by one writer phase regardless of the arrival pattern. This allows all jobs to meet their deadlines in this example, and, in fact, for any arrival pattern of the jobs depicted in Fig. 2.

\subsection{A Phase-Fair Reader-Writer Lock}

While the above example suggests that the properties of phase-fairness can reduce worst-case blocking significantly, to be a viable choice, phase-fair locks must be efficiently implementable on common hardware platforms. In this section, we present a simple and efficient phase-fair RW lock that only depends on hardware support for atomic-add, fetch-and-add, and atomic stores. The algorithm, as given in its entirety in Listing 1, assumes a 32-bit little-endian architecture. However, it can be easily adapted to 16-bit, 64-bit, and big-endian architectures.

Structure. The lock consists of four counters that count the number of issued (rin, win) and completed (rout, wout) read and write requests (lines 1-3 of Listing 1). rin serves multiple purposes: bits 8-31 are used for counting issued read requests, while bit 1 (PRES) is used to signal the presence of unsatisfied write requests and bit $0(P H I D)$ is used to tell consecutive writer phases apart. For efficiency reasons (explained below), bits 2-7 remain unused, as do bits 0-7 of rout for reasons of symmetry. The allocation of bits in rin and rout is illustrated in Fig. 3.

Readers. The reader entry procedure (lines 10-13) works as follows. First, a reader atomically increments rin and observes PRES and PHID (line 12). If no writer is present $(w=0)$, then the reader is admitted immediately (line 13). Otherwise, the reader spins until either of the two writer bits changes: if both bits are cleared, then no writer is present any longer, otherwise - if only PHID toggles but PRES remains unchanged - the beginning of a reader phase has been signaled. The reader exit procedure (lines 15-16) only consists of atomically incrementing rout, which allows a blocked

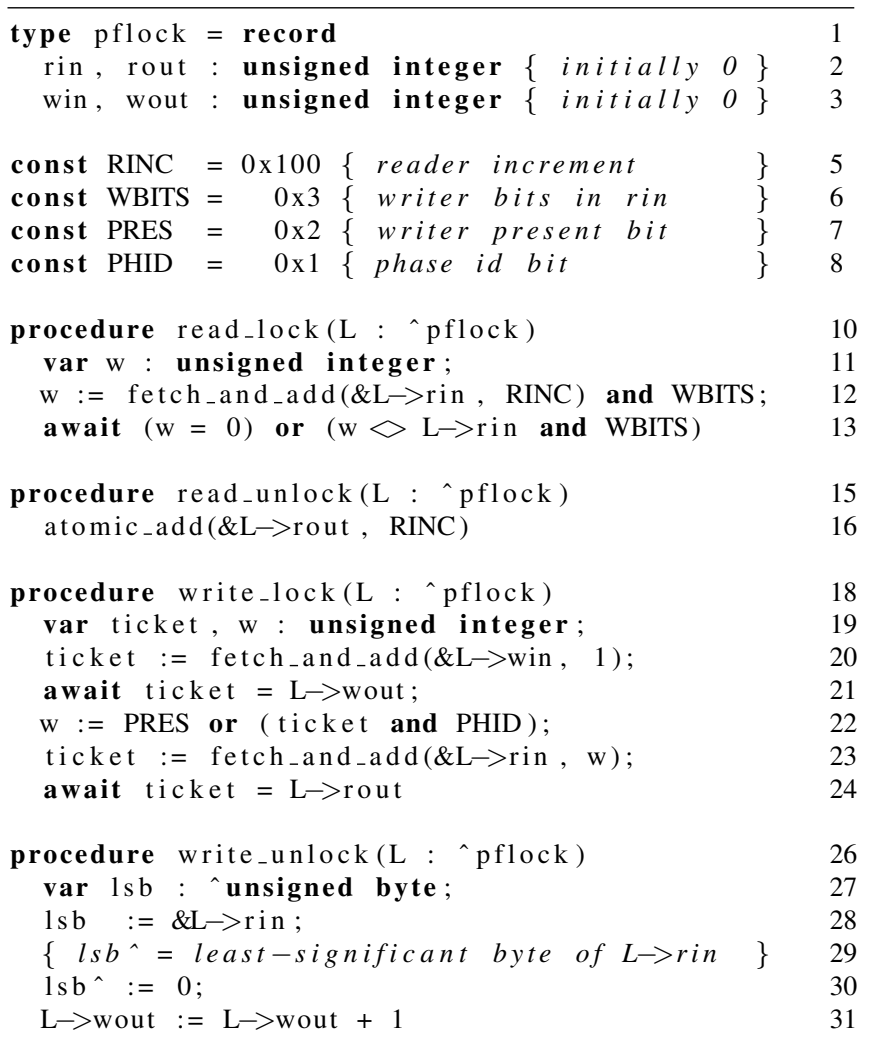

Listing 1: Implementation of a phase-fair spin-based reader-writer lock (algorithm RW-PF).

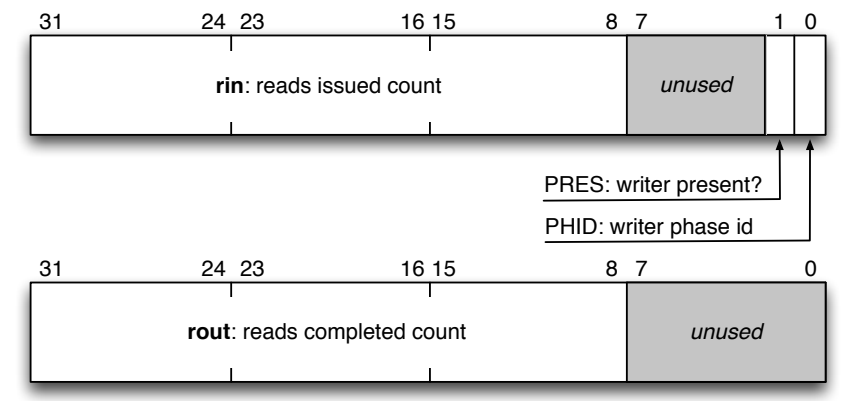

Figure 3: The allocation of bits in the reader entry counter and the reader exit counter (corresponding to Line 2 of Listing 1).

writer to detect when the lock ceases to be held by readers (as discussed below, line 24).

Writers. Similarly to Mellor-Crummey and Scott's simple task-fair RW lock [28], FIFO ordering of writers is realized with a ticket abstraction [27]. The writer entry procedure (lines 18-24) starts by incrementing win in line 20 and waiting for all prior writers to release the lock (line 21). Once a writer is the head of the writer queue (ticket $=$ wout), it atomically sets PRES to one, sets PHID to equal the leastsignificant bit of its ticket, and observes the number of issued read requests (lines 22-23). Note that the least-significant byte of rin equals zero when observed in line 23 since no other writer can be present. Finally, the writer spins until 


\begin{tabular}{llll} 
Algorithm & Name & Complexity & Fairness \\
\hline Mutex Ticket Lock [27] & MX-T & $O(m)$ & task-fair \\
Mutex Queue Lock [27] & MX-Q & $O(1)$ & task-fair \\
Reader-Writer Ticket Lock [28] & RW-T & $O(m)$ & task-fair \\
Reader-Writer Queue Lock [28] & RW-Q & $O(1)$ & task-fair \\
Phase-Fair Lock (Listing 1) & RW-PF & $O(m)$ & phase-fair
\end{tabular}

Table 1: Local-spin locking algorithms considered in this paper. The complexity metric is remote memory references on $m$ cachecoherent processors.

all readers have released the lock before entering its critical section in line 24. The writer exit procedure consists of two steps. First, the beginning off a reader phase is signaled by clearing bits $0-7$ of rin by atomically writing zero to its leastsignificant byte (lines 28-30). Clearing the complete leastsignificant byte instead of just bits 0 and 1 is a performance optimization since writing a byte is usually much faster than atomic read-modify-write instructions on modern hardware architectures. Finally, the writer queue is updated by incrementing wout in line 31 .

Phase id bit. The purpose of $P H I D$ is to avoid a potential race between a slow reader $\left(\mathcal{R}_{1}\right)$ and two writers $\left(\mathcal{W}_{1}\right.$, $\left.\mathcal{W}_{2}\right)$. Assume that $\mathcal{W}_{1}$ holds the lock and that $\mathcal{R}_{1}$ and $\mathcal{W}_{2}$ are blocked. When $\mathcal{W}_{1}$ releases the lock, PRES is cleared (line 30) and wout is incremented (line 31). Subsequently, $\mathcal{W}_{2}$ re-sets PRES (line 23) and waits for $\mathcal{R}_{1}$ to release the lock. Assuming the absence of $P H I D$, if $\mathcal{R}_{1}$ would fail to observe the short window during which PRES is cleared, then it would continue to spin in line 13, waiting for the next writer phase to end. This deadlock between $\mathcal{R}_{1}$ and $\mathcal{W}_{2}$ is avoided by checking PHID: if $\mathcal{R}_{1}$ misses the window between writers, it can still reliably detect the beginning of a reader phase when PHID is toggled.

Size concerns. Overflowing rin, win, rout, and wout is harmless as long as there are at most $2^{32}-1$ concurrent writers and $2^{24}-1$ concurrent readers because the counters are only tested for equality. Note that there can be most $m$ concurrent readers and writers under the FMLP since requests are executed non-preemptively.

In Listing 1, the four counters are defined as four-byte integers each for clarity and performance reasons. However, requiring 16 bytes per lock may be excessive in the context of some memory-constrained applications (e.g., embedded systems). We provide a phase-fair RW lock that only requires 4 bytes and supports up to 127 concurrent readers and writers each in [9].

Implementation in LITMUS ${ }^{\mathrm{RT}}$. In order to realize group locks efficiently, we implemented optimized versions of two local-spin mutex locks and two local-spin RW locks proposed by Mellor-Crummey and Scott [27, 28], as well as our phasefair RW lock, in LITMUS ${ }^{\mathrm{RT}}$, UNC's Linux-derived real-time OS $[13,17]$. As seen in Table 1, these locks are denoted MX$T, M X-Q, R W-T, R W-Q$, and RW-PF respectively.
We implemented each lock on both Intel's x86 and Sun's SPARC V9 architectures. Intel's x86 architecture supports atomic-add and fetch-and-add directly via the add and xadd instructions. On Sun's SPARC V9 architecture, a RISClike design, only compare-and-swap is natively supported and hence both instructions are emulated.

One commonly-used complexity metric for locking algorithms is to count remote memory references (RMR) [2], i.e., on a cache-coherent multiprocessor, locks are classified by how many cache invalidations occur per request under maximum contention. In theory, $O(1)$ locks, e.g., MX-Q, RW$\mathrm{Q}$, should outperform $O(m)$ locks, e.g., MX-T, RW-T, since frequent cache invalidations cause memory bus contention. However, micro benchmarks in which a lock was accessed repeatedly in a tight loop revealed that - on both $\mathrm{x} 86$ and SPARC V9 platforms-the MX-Q and RW-Q locks incur significantly higher overheads than the ticket-based MX-T and $\mathrm{RW}-\mathrm{T}$ locks. The reason is that the $O(1)$ locks inherently require additional slow compare-and-swap instructions. In our micro benchmarks, the positive effects of their lower RMR complexity (i.e., reduced bus traffic) only starts to outweigh the increased entry and exit overheads at unrealistic contention levels, i.e., spinning must comprise a majority of the system's load in order to break even, which is rather unlikely to occur in well-designed real-time systems. Hence, we only consider the MX-T and RW-T locks in the rest of this paper.

\section{Experimental Evaluation}

To compare the various synchronization options discussed above, we determined the HRT and SRT schedulability of randomly-generated task sets under both partitioned and global scheduling assuming the use of MX-T, RW-T, and RWPF group locks. The methodology followed in this study, and our results, are discussed below.

\subsection{Overheads}

In order to capture the impact (or lack thereof) of RW synchronization as accurately as possible, we conducted our study under consideration of real-world system overheads as incurred in LITMUS ${ }^{\mathrm{RT}}$ on a Sun UltraSPARC T1 "Niagara" multicore platform. The Niagara is a 64-bit machine containing eight cores on one chip running at $1.2 \mathrm{GHz}$. Each core supports four hardware threads, for a total of $32 \mathrm{log}$ ical processors. On-chip caches include a 16K (respective, $8 \mathrm{~K}$ ) four-way set associative L1 instruction (respective, data) cache per core, and a shared, unified 3 MB 12-way set associative L2 cache. Our test system is configured with $16 \mathrm{~GB}$ of off-chip main memory. Most relevant system overheads (e.g., scheduling overhead, context-switch overhead, etc.) were already known from a recent study [14] and did not have to be re-determined. Only synchronization-related overheads had to be obtained and are given in Table 2. When determining 


\begin{tabular}{|l|c|c|}
\hline Overhead & Worst-Case & Average-Case \\
\hline task-fair mutex: read/write request & 0.130 & 0.129 \\
\hline task-fair reader-writer lock: read request & 0.160 & 0.157 \\
\hline task-fair reader-writer lock: write request & 0.154 & 0.153 \\
\hline task-fair phase-fair lock: read request & 0.144 & 0.142 \\
\hline task-fair phase-fair lock: write request & 0.180 & 0.178 \\
\hline leaving non-preemptive section & 2.137 & 1.570 \\
\hline
\end{tabular}

Table 2: Worst-case and average-case costs of synchronization overheads (in $\mu s$ ). Based on the methodology explained in detail in [14], the results were obtained by computing the maximum (resp. average) cost of 1,000,000 requests after discarding the top $1 \%$ to remove samples that were disturbed by interrupts and other outliers.

HRT (SRT) schedulability, we assume worst-case (averagecase) overheads (as in [14]).

\subsection{Task Set Generation}

In generating random task sets for conduction schedulability comparisons, task parameters were selected-similar to the approach previously used in $[11,14,15]$ - as follows. Task utilizations were distributed uniformly over $[0.1,0.4]$ and periods were chosen from $[10 \mathrm{~ms}, 100 \mathrm{~ms}]$. We only considered implicit deadlines, i.e., $\mathrm{p}\left(T_{i}\right)=\mathrm{d}\left(T_{i}\right)$, since most G-EDF schedulability tests require this constraint. Task execution costs were calculated based on utilizations and periods. Periods were defined to be integral, but execution costs may be non-integral. Task sets were obtained by generating tasks until a utilization cap (ucap) was reached. We selected these parameter ranges because they correspond to the "medium weight distribution" previously considered in [14]. The effect of choosing heavier or lighter utilization distributions is considered in the discussion of the results below.

Resource sharing. The total number of generated tasks $N$ and the average number of resources per task (res) was used to determine the total number of resources $R=N \cdot$ res. Based on $R$ and the average number of requests per resource per second (contention), the total request density $Q=R \cdot$ contention was computed. Note that request density is a normalized measure similar to task utilization and not necessarily integral. $Q$ was further split based on the ratio of write requests (wratio) into write density, $Q_{w}=Q \cdot$ wratio, and read density, $Q_{r}=Q \cdot(1-$ wratio $)$.

In the next step, we generated read (write) requests and randomly assigned them to tasks until the total request density of the generated read (write) requests equalled $Q_{r}\left(Q_{w}\right)$. Request density is the normalized rate at which a request is issued. ${ }^{4}$ We chose a request period of one unless that would have exceeded $Q_{r}\left(Q_{w}\right){ }^{5}$ Based on the nesting probability (nest), each request contained $d$ levels of nested requests with a probability of $n e s t^{d}$. Resource groups as mandated by the FMLP were computed during request generation. Due to the

\footnotetext{
${ }^{4}$ A request $\mathcal{X}$ assigned to $T_{i}$ has a density of $\frac{1000 \mathrm{~ms}}{\mathrm{p}\left(T_{i}\right) \cdot \mathrm{rp}(\mathcal{X})}$. The factor of $1000 \mathrm{~ms}$ is due to the use of one second as the normalization interval.

${ }^{5}$ Larger request periods are common for low wratios.
}

randomized assignment of requests, some tasks may not request resources at all, and some tasks may both read and write the same resource. However, we ensured that each resource is requested by at least one writer and one reader that are not identical. The duration of requests was distributed uniformly in $[1.0 \mu \mathrm{s}, 15.0 \mu \mathrm{s}]$. This range was chosen to correspond to request durations considered in prior studies on mutex synchronization [11, 15], which in turn were based on durations observed in actual systems [10]. The effect of allowing longer durations is discussed below.

\subsection{Schedulability Tests}

After a task system was generated, its schedulability assuming MX-T, RW-T, and RW-PF group locks was tested as follows. System and synchronization overheads were accounted for by inflating worst-case execution costs and the durations of outermost requests using standard techniques [26]. Per-task bounds on worst-case blocking were computed as detailed in [9], and each task's worst-case execution cost was inflated to account for the corresponding utilization loss due to spinning.

Under G-EDF, a task system was deemed SRT schedulable if the total utilization after inflation did not exceed $m=32$ [19]. Determining whether a task system is hardschedulable under G-EDF is more involved. There now are five major sufficient (but not necessary) HRT schedulability tests for G-EDF [4, 5, 6, 7, 20]. Interestingly, for each of these tests, there exist task sets that are deemed schedulable by it but not the others [5, 7]. Thus, a task system was deemed HRT schedulable under G-EDF if it passed at least one of these five tests.

Under P-EDF, a task system was deemed schedulable if it could be partitioned using the worst-fit decreasing heuristic and the utilization after inflation did not exceed one on any processor (HRT and SRT schedulablity under P-EDF is the same except for the use of worst-case versus averagecase overheads.) [25]. Note that partitioning must precede the blocking-term calculation.

\subsection{Study}

In our study, we assessed SRT/HRT schedulability under both G-EDF and P-EDF while varying five parameters: (i) ucap $\in[1.0,32.0]$, (ii) contention $\in[50,550]$, (iii) wratio $\in[0.01,0.5]$, (iv) nest $\in[0.0,0.5]$, and (v) res $\in$ $[0.1,5.0]$. Each of these parameters was varied over its stated range for all possible choices of the other parameters arising from ucap $\in\{6.0,9.0,12.0\}$ for HRT and ucap $\in$ $\{15.0,18.0,21.0\}$ for SRT, contention $\in\{100,250,400\}$, wratio $\in\{0.05,0.2,0.35\}$, nest $\in\{0.05,0.2,0.35\}$, and res $\in\{0.5,2.0,3.5\}$, under both SRT and HRT and both G-EDF and P-EDF scheduling. Sampling points were chosen such that the sampling density is high in areas where curves change rapidly. For each sampling point, we gener- 
ated (and tested for schedulability) 50 task sets, for a total of over 1,600,000 tested task sets.

Trends. It is clearly not feasible to present all 1,620 resulting graphs. However, the results show clear trends. We begin by making some general observations concerning these trends. Below, we consider a few specific graphs that support these observations.

In the majority of the tested scenarios, RW-PF locks were clearly the best-performing algorithm. MX-T locks were preferable in only 23 of the 1,620 tested scenarios (12 under G-EDF, 11 under P-EDF). Due to the wide range of parameter values considered, some parameter combinations did not exhibit discernible trends since they were either "too easy" (low contention, res, ucap) or "too hard" (high ucap, contention, wratio, nest) - either (almost) all or none of the task sets were schedulable in these scenarios regardless of the group lock type. Where RW synchronization was preferable to mutual exclusion, RW-T locks never outperformed RW-PF locks.

Generally speaking, RW-PF locks were usually more resilient to increases in contention, res, ucap, nest, and wratio, i.e., they exhibited higher schedulability than the other two choices under increasingly adverse conditions. Hence, it is more illuminating to consider the two exceptions: (i) under which conditions are MX-T locks preferable to RWPF locks (and why), and (ii) under which conditions do RW-T locks perform as well as RW-PF locks? Regarding (i), all 23 cases exhibit a combination of high request density, deep and frequent nesting, and many writers (wratio $\geq 0.35$ ). Hence, writer blocking, which is not improved by RW locks, becomes the dominating performance factor. This explains why RWPF (and RW-T) locks do not perform better than MX-T locks in some cases, but why do they perform worse? The reason is additional pessimism in the worst-case blocking analysis of RW-PF locks that is only triggered by scenarios that involve many writers and frequent, long requests (see [9]). The answer to (ii) reinforces the intuition that task-fair locks are very sensitive to the number of concurrent writers: all scenarios in which RW-T locks perform as well as RW-PF locks (and in which RW synchronization is preferable to mutual exclusion) exhibit a very low write density (wratio $=0.05$ ). However, the inverse is not true: there are scenarios in which RW-PF locks clearly perform better than RW-T locks (in terms of schedulability) where wratio $=0.05$.

Example graphs. Insets (a)-(j) of Fig. 4 display ten selected graphs that illustrate the above trends. In order to show a wide variety of scenarios, we chose to exhibit one HRT schedulability result under P-EDF (left column) and one SRT schedulability result under G-EDF (right column) for each of the five parameters that we varied (rows).

Insets (a)-(b) show schedulability as a function of ucap. In both cases, RW-PF locks yield better schedulability: under P-EDF (resp., G-EDF), with MX-T and RW-T locks, per- formance starts to degrade ucap $\approx 8$ (resp., ucap $\approx 13$ ), whereas RW-PF locks can sustain high schedulability until ucap $\approx 10$ (resp., ucap $\approx 16$ ). Note that with only $20 \%$ writes RW-T locks perform almost as badly as MX$\mathrm{T}$ locks. Insets (c)-(d) show schedulability as a function of contention. For both P-EDF and G-EDF, RW-PF locks can sustain almost twice as much contention as either MX-T or RW-T locks before performance degrades. This highlights the significance of the RW-PF lock $O(1)$ bound on read blocking under high contention. Again, RW-T locks offer little advantage over MX-T locks. Insets (e)-(f) show schedulability as a function of wratio. Inset (e) illustrates under which conditions MX-T locks are (partially) preferable. Due to high contention, nest, and res, RW-PF locks start to perform worse than MX-T locks when wratio $\geq 0.35$ due to pessimism in the analysis with regard to high number of writers. In contrast, inset (f) shows a more representative scenario in which RW-PF locks can sustain high schedulability until wratio $\approx 0.3$, whereas $\mathrm{RW}-\mathrm{T}$ locks are only usable when there are virtually no writes even with moderate nest and res. Insets ( $\mathrm{g})$-(h) show schedulability as a function of nest. In both cases, RW-PF locks perform significantly better than either MX-T and RW-T locks as resource groups become fewer in number and larger, even under high res $(\mathrm{P}$ EDF case) and high contention (G-EDF case). Finally, insets (i)-(j) show schedulability as a function of res. Once again, RW-PF locks can sustain significantly higher schedulability and RW-T locks perform only little better than MX-T locks.

Varying weights, periods, and request lengths. With lighter utilization distributions, the differences between the locks would be magnified since the number of tasks increases and bounds on worst-case blocking become larger relative to the average worst-case execution cost. Similarly, longer request durations would also magnify differences in performance. Lengthening periods causes a decrease in average perrequest density, which in turn causes the number of requests per task to increase. Further, the interval for which interference needs to be analyzed increases [9]. In both cases, the bounds on worst-case blocking would be more pessimistic, and hence differences in performance would be magnified.

\section{Conclusion}

We have presented the first analysis of reader-writer locking in shared-memory multiprocessor real-time systems and demonstrated that preference- and task-fair locks can be problematic in such systems. We also have proposed phase-fair locks, an alternative lock design with asymptotically lower worstcase read blocking. Our experiments revealed that (in terms of schedulability) phase-fair locks are almost always the best choice_-oftentimes by a significant margin.

In future work, we would like to extend the RW-FMLP 


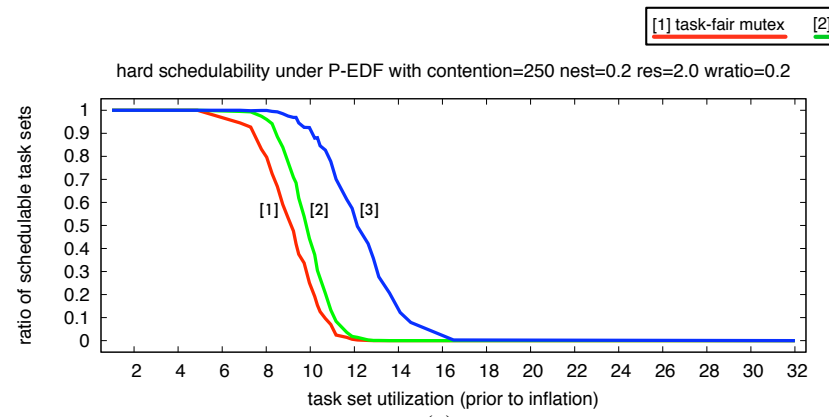

(a)

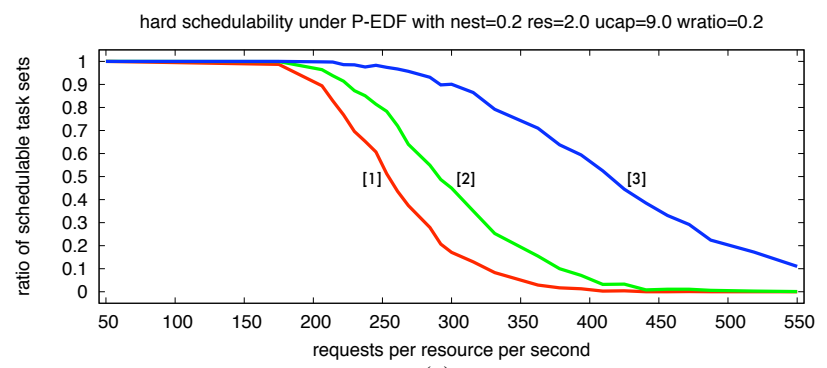

(c)

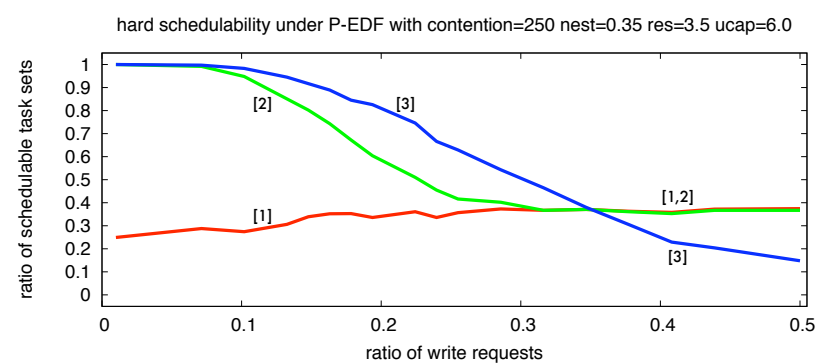

(e)

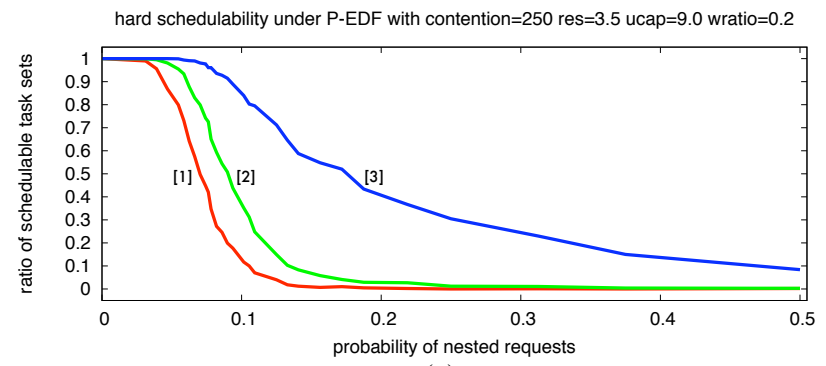

(g)

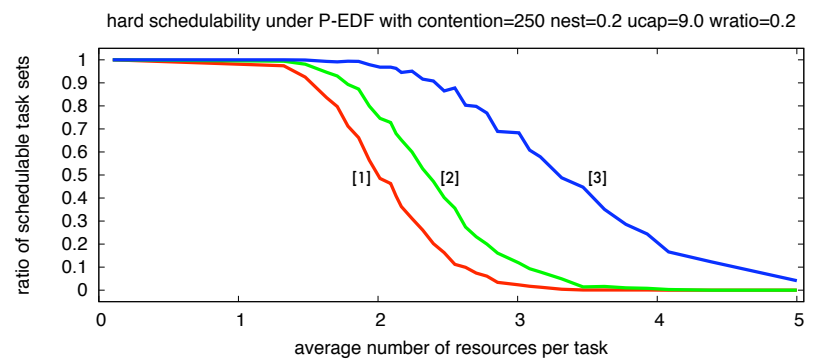

(i)

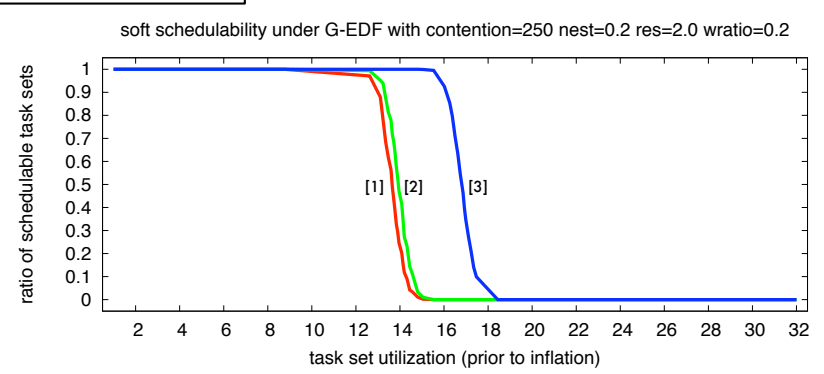

(b)

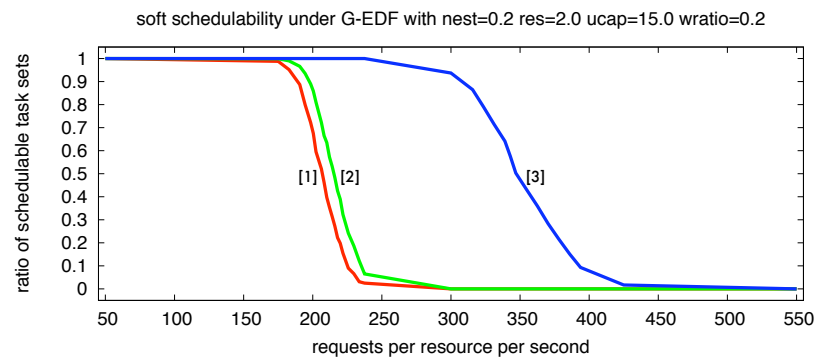

(d)

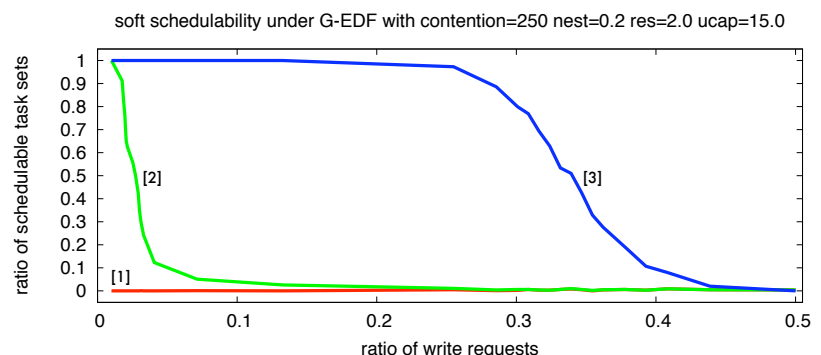

(f)

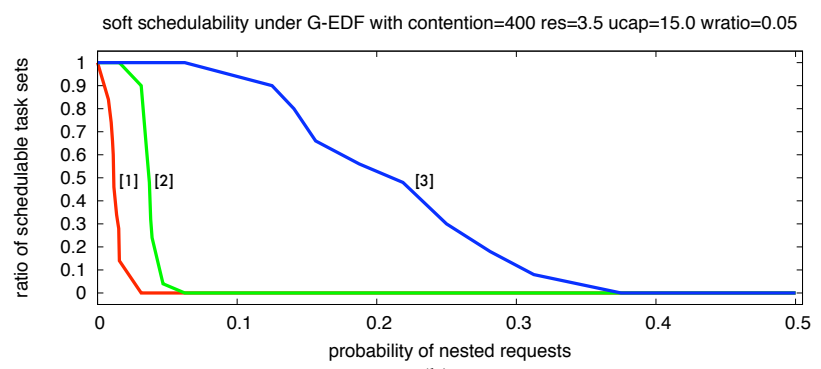

(h)

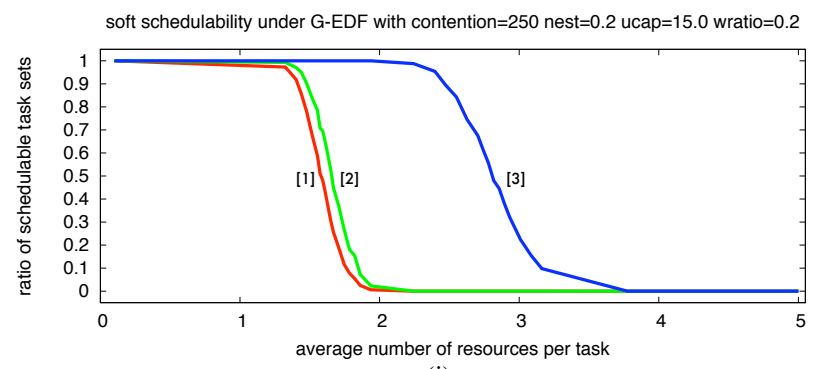

(j)

Figure 4: Schedulability (the fraction of generated task systems deemed schedulable) as a function of (a)-(b) task system utilization (ucap), (c)-(d) average requests per resource per second (contention), (e)-(f) ratio of write requests (wratio), (g)-(h) probability of nested requests (nest), (i)-(j) average number of resources per task (res). The left column shows hard schedulability under P-EDF; the right right column shows soft schedulability under G-EDF. The $y$-axis of each graph gives the fraction of successfully-scheduled task sets. The scenario considered in each graph is indicated above that graph. 
to support suspension-based real-time reader-writer synchronization. Further, we would like to develop a phasefair reader-writer lock with $O(1)$ RMR complexity and reevaluate the performance of reader-writer lock choices in the absence of shared, coherent caches.

\section{References}

[1] J. Anderson and P. Holman. Efficient pure-buffer algorithms for real-time systems. In Proc. of the Seventh International Conference on Real-Time Systems and Applications, pp. 5764, 2000.

[2] J. Anderson, Y. Kim, and T. Herman. Shared-memory mutual exclusion: major research trends since 1986. Distributed Computing, 16(2-3):75-110, 2003.

[3] T. Baker. Stack-based scheduling for realtime processes. RealTime Systems, 3(1):67-99, 1991.

[4] T. Baker. Multiprocessor EDF and deadline monotonic schedulability analysis. In Proc. of the 24th IEEE Real-Time Systems Symposium, pp. 120-129, 2003.

[5] Sanjoy Baruah. Techniques for multiprocessor global schedulability analysis. In Proc. of the 28th IEEE International RealTime Systems Symposium, pp. 119-128, 2007.

[6] Marko Bertogna, Michele Cirinei, and Giuseppe Lipari. Improved schedulability analysis of edf on multiprocessor platforms. In Proc. of the 17th Euromicro Conference on Real-Time Systems, pp. 209-218, 2005.

[7] Marko Bertogna, Michele Cirinei, and Giuseppe Lipari. Schedulability analysis of global scheduling algorithms on multiprocessor platforms. IEEE Transactions on Parallel and Distributed Systems, 99(1):(to appear), 2008.

[8] A. Block, H. Leontyev, B. Brandenburg, and J. Anderson. A flexible real-time locking protocol for multiprocessors. In Proc. of the 13th IEEE International Conference on Embedded and Real-Time Computing Systems and Applications, pp. 47-57, 2007.

[9] B. Brandenburg and J. Anderson. Reader-writer synchronization for shared-memory multiprocessor real-time systems (extended version), 2009.

Available at http://www.cs.unc.edu/ anderson/papers.html.

[10] B. Brandenburg and J. Anderson. Feather-trace: A light-weight event tracing toolkit. In Proc. of the Third International Workshop on Operating Systems Platforms for Embedded Real-Time Applications, pp. 20-27, 2007.

[11] B. Brandenburg and J. Anderson. A comparison of the M-PCP, D-PCP, and FMLP on LITMUS ${ }^{\mathrm{RT}}$. In Proc. of the 12th International Conference On Principles Of Distributed Systems, pp. 105-124, 2008.

[12] B. Brandenburg and J. Anderson. An implementation of the PCP, SRP, D-PCP, M-PCP, and FMLP real-time synchronization protocols in LITMUS ${ }^{\mathrm{RT}}$. In Proc. of the 14th IEEE RealTime and Embedded Technology and Applications Symposium, pp. 185-194, 2008.

[13] B. Brandenburg, A. Block, J. Calandrino, U. Devi, H. Leontyev, and J. Anderson. LITMUS ${ }^{\mathrm{RT}}$ : A status report. In Proc. of the 9th Real-Time Linux Workshop, pp. 107-123, 2007.

[14] B. Brandenburg, J. Calandrino, and J. Anderson. On the scalability of real-time scheduling algorithms on multicore plat- forms: A case study. In Proc. of the 29th IEEE Real-Time Systems Symposium, pp. 157-169, 2008.

[15] B. Brandenburg, J. Calandrino, A. Block, H. Leontyev, and J. Anderson. Synchronization on real-time multiprocessors: To block or not to block, to suspend or spin? In Proc. of the 14th IEEE Real-Time and Embedded Technology and Applications Symposium, pp. 342-353, 2008.

[16] J. Calandrino, J. Anderson, and D. Baumberger. A hybrid realtime scheduling approach for large-scale multicore platforms. In Proc. of the 19th Euromicro Conference on Real-Time Systems, pp. 247-256, 2007.

[17] J. Calandrino, H. Leontyev, A. Block, U. Devi, and J. Anderson. LITMUS ${ }^{\mathrm{RT}}$ : A testbed for empirically comparing realtime multiprocessor schedulers. In Proc. of the 27th IEEE RealTime Systems Symposium, pp. 111-123, 2006.

[18] P. Courtois, F. Heymans, and D. Parnas. Concurrent control with "readers" and "writers". Communications of the ACM, 14(10):667-668, 1971.

[19] U. Devi and J. Anderson. Tardiness bounds under global EDF scheduling on a multiprocessor. Real-Time Systems, 38(2):133189, 2008.

[20] J. Goossens, S. Funk, and S. Baruah. Priority-driven scheduling of periodic task systems on multiprocessors. Real-Time Systems, 25(2-3):187-205, 2003.

[21] P. Gore, I. Pyarali, C. Gill, and D. Schmidt. The design and performance of a real-time notification service. In Proc. of the 10th IEEE Real-Time and Embedded Technology and Applications Symposium, pp. 112-120, 2004.

[22] W. Hsieh and W. Weihl. Scalable reader-writer locks for parallel systems. In Proc. of the 6th International Parallel Processing Symposium, pp. 656-659, 1992.

[23] O. Krieger, M. Stumm, R. Unrau, and J. Hanna. A fair fast scalable reader-writer lock. In Proc. of the 1993 International Conference on Parallel Processing, pp. 201-204, 1993.

[24] Q. Li and C. Yao. Real-Time Concepts for Embedded Systems. CMP Books, 2003.

[25] C. Liu and J. Layland. Scheduling algorithms for multiprogramming in a hard real-time environment. Journal of the ACM, 30:46-61, 1973

[26] J. Liu. Real-Time Systems. Prentice Hall, 2000.

[27] J. Mellor-Crummey and M. Scott. Algorithms for scalable synchronization on shared-memory multiprocessors. ACM Transactions on Computer Systems, 9(1):21-65, 1991.

[28] J. Mellor-Crummey and M. Scott. Scalable reader-writer synchronization for shared-memory multiprocessors. In Proc. of the 3rd ACM SIGPLAN symposium on principles and practice of parallel programming, pp. 106-113, 1991.

[29] M. Musial, V. Remuß, C. Deeg, and G. Hommel. Embedded system architecture of the second generation autonomous unmanned aerial vehicle MARVIN MARK II. In Proc. of the 7th International Workshop on Embedded Systems-Modeling, Technology and Applications, pp. 101-110, 2006.

[30] R. Rajkumar. Synchronization In Real-Time Systems - A Priority Inheritance Approach. Kluwer Academic Publishers, 1991.

[31] M. Reiman and P. Wright. Performance analysis of concurrentread exclusive-write. In Proc. of the 1991 ACM SIGMETRICS Conference on Measurement and modeling of computer systems, pp. 168-177, 1991. 


\section{A Bounding Blocking Time}

Blocking is any delay encountered by a job that would not have arisen if all tasks were independent. The maximum blocking duration must be bounded and accounted for when testing schedulability. In this appendix, we derive such bounds for the scheduling and synchronization algorithms considered in this paper. In this section, we first give an overview over our notation (A.1), discuss the common analysis approach (A.2), and provide basic definitions (A.3). In the following sections, we then derive bounds on direct blocking for fair mutex locks (A.5), task-fair RW locks (A.6), and phase-fair RW locks (A.7). Finally, the impact of arrival blocking is considered (A.8).

\section{A.1 Notation}

For convenience, some of the earlier definitions are repeated.

$\begin{array}{ll}m & \text { The number of processors. } \\ T_{i} & \text { The } i \text { th of } n \text { sporadic tasks (with implicit deadlines). } \\ T_{i}^{j} & \text { The } j \text { th job of } T_{i} . \\ \mathrm{r}\left(T_{i}^{j}\right) & \text { The release time of } T_{i}^{j} . T_{i}^{j} \text { is pending from } \mathrm{r}\left(T_{i}^{j}\right) \text { until it completes. } \\ \mathrm{p}\left(T_{i}\right) & \text { The period of } T_{i} \text {. } \\ \mathrm{d}\left(T_{i}\right) & \text { The relative deadline of } T_{i} . \\ \mathrm{c}\left(T_{i}\right) & \text { The completion-time requirement for task } T_{i} . \\ & \text { Under hard real-time scheduling, } \mathrm{c}\left(T_{i}\right)=\mathrm{d}\left(T_{i}\right) . \\ & \text { Hence, with implicit deadlines and hard real-time scheduling, } \mathrm{c}\left(T_{i}\right)=\mathrm{p}\left(T_{i}\right) . \\ \ell & \text { Under soft real-time scheduling, } \mathrm{c}\left(T_{i}\right) \text { is bounded by } \mathrm{d}\left(T_{i}\right) \text { and } T_{i} \text { 's tardiness bound. } \\ \operatorname{grp}(\ell) & \text { A resource. } \\ \mathcal{R} & \text { The resource group that contains } \ell . \\ \mathcal{W} & \text { A read request for a resource. } \\ \mathcal{X} & \text { A write request for a resource. } \\ \operatorname{res}(\mathcal{X}) & \text { A request for a resource (either read or write). } \\ \operatorname{tsk}(\mathcal{X}) & \text { The resource requested by } \mathcal{X} . \\ \operatorname{rp}(\mathcal{X}) & \text { The task that issues } \mathcal{X} . \\ |\mathcal{X}| & \text { The request period of } \mathcal{X} \text { (see below). } \\ & \text { An upper bound on the maximum duration for which res }(\mathcal{X}) \text { will be held. } \\ \operatorname{reads}\left(T_{i}\right) & \text { Does not include blocking due to } \mathcal{X} . \\ \operatorname{writes}\left(T_{i}\right) & \text { A set of outermost read requests that bounds the worst-case reading behavior of any one job of } T_{i} . \\ & \text { A set of outermost write requests that bounds the worst-case writing behavior of any one job of } T_{i} .\end{array}$

For notational convenience, we assume $\max (\emptyset)=0$. Under both P-EDF and P-SP scheduling, we assume that the task set has been successfully partitioned prior to computing blocking terms and let $\mathrm{P}\left(T_{i}\right)$ denote the processor $(1, \ldots, m)$ to which $T_{i}$ is assigned. Under P-SP scheduling, we further assume that tasks are indexed by decreasing priority (i.e., $T_{i}$ has higher priority than $T_{j}$ iff $i<j$ ) and that priorities are unique.

Two requests $\mathcal{X}_{1}$ and $\mathcal{X}_{2}$ (either read or write) interfere with each other if $\operatorname{tsk}\left(\mathcal{X}_{1}\right) \neq \operatorname{tsk}\left(\mathcal{X}_{2}\right), \operatorname{grp}\left(\operatorname{res}\left(\mathcal{X}_{1}\right)\right)=$ $\operatorname{grp}\left(\operatorname{res}\left(\mathcal{X}_{2}\right)\right)$, and the issuing jobs can potentially execute concurrently.

\section{A.2 Approach}

The maximum number of times that a job $T_{i}^{j}$ is directly blocked can be bounded by the minimum of both the number of times that $T_{i}^{j}$ issues outermost requests and the number of times that remote jobs issue interfering requests. ${ }^{6}$ Bounding the maximum duration of direct blocking is hence a two-step process: first, the set of possibly-interfering remote jobs must be derived (this varies by scheduling algorithm); and second, the worst-case pattern of interfering requests that can delay $T_{i}^{j}$ must be derived (this varies by synchronization protocol). Since groups are independent, analysis can be done on a group-by-group basis. Given a bound $\operatorname{dbg}\left(T_{i}, g\right)$ on the maximum duration of direct blocking incurred by one job of $T_{i}$ due to requests issued for resources

\footnotetext{
${ }^{6}$ Note that this is a direct consequence of using FIFO queuing: if $T_{i}^{j}$ makes only one request for a resource $\ell$, then it can be directly blocked at most once by jobs on each remote processor no matter how many conflicting requests are issued while $T_{i}^{j}$ is pending. In contrast, if wait queues were ordered by priority, then a single request by $T_{i}^{j}$ could lead to $T_{i}^{j}$ being blocked multiple times by each remote higher-priority task.
} 


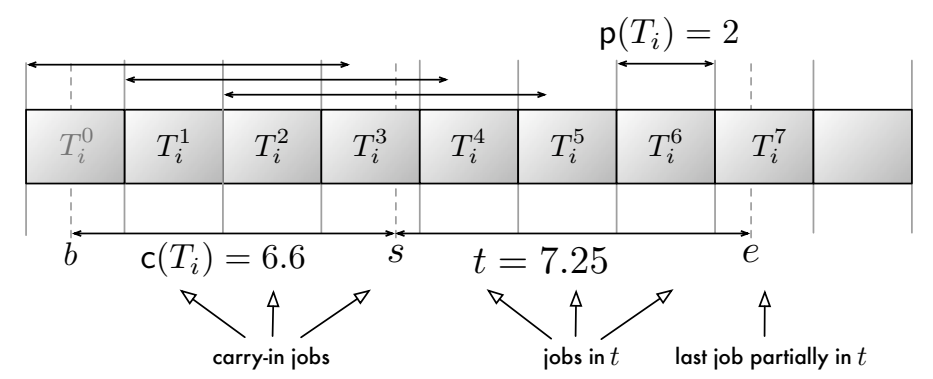

Figure 5: An illustration of (2). At most seven jobs of $T_{i}$ with $\mathrm{p}\left(T_{i}\right)=2$ and $\mathrm{c}\left(T_{i}\right)=6.6$ can be pending in any interval $[s, e)$ of length $e-s=t=7.25$ (illustration drawn to scale). Only jobs released at or after $b=s-\mathrm{c}\left(T_{i}\right)$ and before $e$ can be pending in $[s, e]$. In the worst case (i.e., with periodic arrivals and jobs completing as late as possible), jobs $T_{i}^{1}, T_{i}^{2}$, and $T_{i}^{3}$ carry execution into $[s, e]$. Jobs $T_{i}^{4}, T_{i}^{5}$, and $T_{i}^{6}$ are released and complete in $[s, e]$, and $T_{i}^{7}$ is released before $e$. Note that moving the start of the interval $s$ to an earlier point such that $\mathrm{r}\left(T_{i}^{0}\right) \geq b$ causes the last counted job $T_{i}^{7}$ to no longer be pending within $\left[s\right.$, e), i.e. $e<\mathrm{r}\left(T_{i}^{7}\right)$. Hence $\operatorname{maxjobs}\left(T_{i}, 7.25\right)=\left\lceil\frac{13.85}{2.0}\right\rceil=7$.

in resource group $g$, a bound on total direct blocking can be obtained summing over the bounds on blocking for each group that $T_{i}$ accesses, i.e.,

$$
\mathrm{DB}\left(T_{i}\right)=\sum_{g \in x \operatorname{xgps}\left(T_{i}\right)} \operatorname{dbg}\left(T_{i}, g\right),
$$

where $\operatorname{xgrps}\left(T_{i}\right)$ is the set of resource groups that $T_{i}$ accesses (see Eq. (27)). Specific bounds on $d b g\left(T_{i}, g\right)$ are derived in Secs. A.5, A.6, and A.7.

Non-preemptive execution of lower-priority jobs must also be taken into account since this may cause arrival blocking. Bounds on arrival blocking, which vary by scheduling algorithm, are given in Sec. A.8.

\section{A.3 Basic Definitions}

In this subsection, we define terms for recurring concepts in the derivation of the blocking terms. The maximum number of jobs of $T_{i}$ that can execute in an interval of length $t$ given by

$$
\operatorname{maxjobs}\left(T_{i}, t\right)=\left\lceil\frac{t+\mathrm{c}\left(T_{i}\right)}{\mathrm{p}\left(T_{i}\right)}\right\rceil,
$$

as illustrated in Fig. 5. Similarly, based on the concept of a request period, the maximum number of times that a request $\mathcal{X}$ can be issued in an interval of length $t$ is given by

$$
\operatorname{maxreqs}(\mathcal{X}, t)=\left\lceil\frac{\text { maxjobs }(\mathrm{tsk}(\mathcal{X}), t)}{\operatorname{rp}(\mathcal{X})}\right\rceil .
$$

The set of read requests issued by a task $T_{i}$ that interfere with requests for resources in group $g$ is given by

$$
\operatorname{rconflict}\left(T_{i}, g\right)=\left\{\mathcal{R} \mid \mathcal{R} \in \operatorname{reads}\left(T_{i}\right) \wedge \operatorname{grp}(\operatorname{res}(\mathcal{R}))=g\right\}
$$

Similarly, the set of interfering writes is defined as

$$
\operatorname{wconflict}\left(T_{i}, g\right)=\left\{\mathcal{W} \mid \mathcal{W} \in \operatorname{writes}\left(T_{i}\right) \wedge \operatorname{grp}(\operatorname{res}(\mathcal{W}))=g\right\}
$$

Next, we define read/write/request interference, a central concept in the derivation of the blocking terms. The worst-case read interference of task $T_{i}$ affecting group $g$ during an interval of length $t$, denoted as rif $\left(T_{i}, g, t\right)$, is a set of read requests that represents the worst-case sequence of read requests that can be issued by $T_{i}$ over any interval of length $t$, i.e., the sequence of requests created if jobs of $T_{i}$ arrive periodically and issue maximum-duration requests as often as possible (respecting both task and request periods). Note that requests can be issued repeatedly if $t$ is sufficiently large; hence rif $\left(T_{i}, g, t\right)$ is a multiset. (The multiset is constructed by tagging individual requests.)

$$
\operatorname{rif}\left(T_{i}, g, t\right)=\left\{(\mathcal{R}, k) \mid \mathcal{R} \in \operatorname{rconflict}\left(T_{i}, g\right) \wedge k \in\{1, \ldots, \operatorname{maxreqs}(\mathcal{R}, t)\}\right\} .
$$


Likewise, we define write interference as

$$
\text { wif }\left(T_{i}, g, t\right)=\left\{(\mathcal{W}, k) \mid \mathcal{W} \in \operatorname{wconflict}\left(T_{i}, g\right) \wedge k \in\{1, \ldots, \operatorname{maxreqs}(\mathcal{W}, t)\}\right\}
$$

and request interference as

$$
\operatorname{xif}\left(T_{i}, g, t\right)=\left\{(\mathcal{X}, k) \mid \mathcal{X} \in \operatorname{rconflict}\left(T_{i}, g\right) \cup \operatorname{wconflict}\left(T_{i}, g\right) \wedge k \in\{1, \ldots, \operatorname{maxreqs}(\mathcal{X}, t)\}\right\} .
$$

Let $A=\left\{\left(\mathcal{X}_{i}, y_{i}\right)\right\}$ be a (finite) multiset of requests, let $A^{\geq}$denote $A$ ordered by non-decreasing request duration, and let $A_{\bar{k}}^{\geq}$ denote the $k$ th item in $A^{\geq}$(if it exists), i.e., $A^{\geq}$is the list obtained by sorting $A$ with the following relation:

$$
(\mathcal{X}, y) \geq\left(\mathcal{X}^{\prime}, y^{\prime}\right) \Leftrightarrow|\mathcal{X}| \geq\left|\mathcal{X}^{\prime}\right|
$$

We define the set of the $l$ longest requests in $A$ as

$$
\text { longest }(l, A)=\left\{(\mathcal{X}, y) \mid(\mathcal{X}, y)=A_{\bar{k}}^{\geq} \wedge k \in\{1, \ldots, \min (l,|A|)\}\right\}
$$

and the sum of their durations as

$$
\operatorname{total}(l, A)=\sum_{(\mathcal{X}, y) \in \text { longest }(l, A)}|\mathcal{X}| .
$$

The set of the $|A|-l$ shortest requests in $A$ is denoted as

$$
\operatorname{rest}(l, A)=A \backslash \text { longest }(l, A) .
$$

In the derivation of the blocking terms, two subsets of tasks are of particular interest. The set of all tasks but a given task $T_{i}$ is given by

$$
\operatorname{others}(i)=\left\{T_{x} \mid x \in\{1, \ldots, n\} \wedge x \neq i\right\} .
$$

Under partitioning, the set of tasks assigned to processor $p$ is given by

$$
\operatorname{partition}(p)=\left\{T_{i} \mid i \in\{1, \ldots, n\} \wedge \mathrm{P}\left(T_{i}\right)=p\right\} .
$$

Given a set of tasks $S$, we denote the multiset of the $l$ longest potentially interfering (with respect to group $g$ ) read requests of each task during $t$ as

$$
\operatorname{rifs}(S, g, t, l)=\bigcup_{T_{i} \in S} \text { longest }\left(l, \operatorname{rif}\left(T_{i}, g, t\right)\right) .
$$

Analogously, we define

$$
\text { wifs }(S, g, t, l)=\bigcup_{T_{i} \in S} \text { longest }\left(l, w i f\left(T_{i}, g, t\right)\right)
$$

with respect to write interference and

$$
\operatorname{xifs}(S, g, t, l)=\bigcup_{T_{i} \in S} \text { longest }\left(l, x i f\left(T_{i}, g, t\right)\right)
$$

with respect to request interference. In the case of partitioned scheduling, we define the set of the $l$ longest interfering read requests on each remote processor as

$$
\operatorname{pwifs}\left(T_{i}, g, t, l\right)=\bigcup_{\substack{p=1 \\ p \neq \mathrm{P}\left(T_{i}\right)}}^{m} \text { longest }(l, \text { wifs }(\operatorname{partition}(p), g, t, l)) .
$$

Again, we define analogously

$$
\operatorname{prifs}\left(T_{i}, g, t, l\right)=\bigcup_{\substack{p=1 \\ p \neq \mathrm{P}\left(T_{i}\right)}}^{m} \operatorname{longest}(l, \operatorname{rifs}(\operatorname{partition}(p), g, t, l))
$$


with respect to write interference and

$$
\operatorname{pxifs}\left(T_{i}, g, t, l\right)=\bigcup_{\substack{p=1 \\ p \neq \mathrm{P}\left(T_{i}\right)}}^{m} \text { longest }(l, x i f s(\operatorname{partition}(p), g, t, l))
$$

with respect to request interference. The longest non-preemptive section of a set of tasks $S$ is given by

$$
\operatorname{maxnp}(S)=\max \left(\left\{|\mathcal{X}| \mid T_{i} \in S \wedge \mathcal{X} \in \operatorname{reads}\left(T_{i}\right) \cup \operatorname{writes}\left(T_{i}\right)\right\}\right) .
$$

The maximum number of read requests for resources in group $g$ issued by any job of $T_{i}$ is denoted as

$$
\left.\operatorname{rcount}\left(T_{i}, g\right)=\mid\left\{\mathcal{R} \mid \mathcal{R} \in \operatorname{reads}\left(T_{i}\right)\right) \wedge g=\operatorname{grp}(\operatorname{res}(\mathcal{R}))\right\} \mid ;
$$

likewise

$$
\left.\operatorname{wcount}\left(T_{i}, g\right)=\mid\left\{\mathcal{W} \mid \mathcal{W} \in \operatorname{writes}\left(T_{i}\right)\right) \wedge g=\operatorname{grp}(\operatorname{res}(\mathcal{W}))\right\} \mid
$$

and

$$
x \operatorname{count}\left(T_{i}, g\right)=\operatorname{rcount}\left(T_{i}, g\right)+\operatorname{wcount}\left(T_{i}, g\right) .
$$

The set of groups that are subject to read requests issued by $T_{i}$ is given by

$$
\operatorname{rgrps}\left(T_{i}\right)=\left\{\operatorname{grp}(\mathcal{R}) \mid \mathcal{R} \in \operatorname{reads}\left(T_{i}\right)\right\}
$$

likewise

$$
\operatorname{wgrps}\left(T_{i}\right)=\left\{\operatorname{grp}(\mathcal{W}) \mid \mathcal{W} \in \operatorname{writes}\left(T_{i}\right)\right\}
$$

and

$$
\operatorname{xgrps}\left(T_{i}\right)=\operatorname{rgrps}\left(T_{i}\right) \bigcup \operatorname{wgrps}\left(T_{i}\right) .
$$

\section{A.4 Lock Properties}

Next, we establish the fundamental lock properties that we require to derive bounds on the worst-case duration of blocking.

Lemma 1. Let $b$ the number of reader and writer phases that block a request $\mathcal{X}$ (either read or write) before it is satisfied. If contending requests are satisfied in FIFO order and if requests are executed non-preemptively, then $b \leq m-1$.

Proof. Since requests are satisfied in FIFO order, later arriving requests cannot block $\mathcal{X}$. Hence, only requests that were already issued but not yet complete when $\mathcal{X}$ was issued can block $\mathcal{X}$. In the worst case, because a job is not preempted while executing a request, at most one job on every other processor can have issued a request that blocks $\mathcal{X}$.

Lemma 2. Let b the total number of reader and writer phases that block outermost requests (either read or write) issued by $T_{i}^{j}$ for resources in group $g$. If $T_{i}^{j}$ 's requests for resources in $g$ interfere with at most $w$ write requests and with at most $r$ read requests, then $b \leq r+w$.

Proof. Trivial, since every interfering request can block a request of $T_{i}^{j}$ only once.

Lemma 3. Let $c$ be the number of outermost requests (either read or write) that a job $T_{i}^{j}$ issues for resources in group $g$. If contending requests are satisfied in FIFO order, then at most c interfering outermost requests issued by jobs of a task $T_{x}$ can block $T_{i}^{j}$.

Proof. By contradiction. If more than $c$ outermost requests issued by jobs of $T_{x}$ (for resources in $g$ ) block $T_{i}^{j}$, then at least one outermost request $\mathcal{X}^{i}$ issued by $T_{i}^{j}$ was blocked by at least two outermost requests $\left(\mathcal{X}_{1}^{x}, \mathcal{X}_{2}^{x}\right)$ of jobs of $T_{x}$. Because both $\mathcal{X}_{1}^{x}$ and $\mathcal{X}_{2}^{x}$ blocked $\mathcal{X}^{i}$, neither of the two was complete when $\mathcal{X}^{i}$ was issued, but both were complete by the time that $\mathcal{X}^{i}$ was satisfied. Without loss of generality, assume that $\mathcal{X}_{1}^{x}$ was issued prior to $\mathcal{X}_{2}^{x}$. Because tasks are sequential (and because both $\mathcal{X}_{1}^{x}$ and $\mathcal{X}_{2}^{x}$ are outermost), $\mathcal{X}_{1}^{x}$ must have been complete when $\mathcal{X}_{2}^{x}$ was issued. Hence $\mathcal{X}_{2}^{x}$ was issued after $\mathcal{X}^{i}$ was issued, but satisfied before $\mathcal{X}^{i}$ was satisfied. This contradicts the assumption that contending requests are satisfied in FIFO order. 


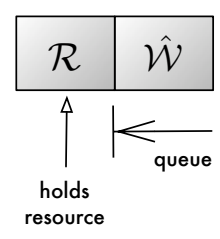

(a)

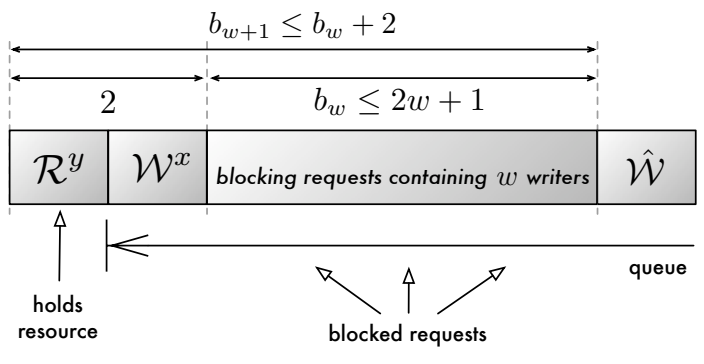

(b)

Figure 6: Illustration of Lemma 4 for the case $c_{\mathcal{W}}=1$ wherein a job $T_{i}^{j}$ issues only one write request $\hat{\mathcal{W}}$. (a) Base case $w=0$ : at most one reader phase can block $\hat{\mathcal{W}}$. (b) Induction step: allowing one additional write request $\mathcal{W}^{x}$ increases the maximum number of blocking phases by at most two.

Lemma 4. Let $c_{\mathcal{R}}$ be the number of outermost read requests and let $c_{\mathcal{W}}$ be the number of outermost write requests that a job $T_{i}^{j}$ issues for resources in group $g$, and let $b_{w}$ be the total number of reader and writer phases that block $T_{i}^{j}$ across all outermost requests for resources in $\mathrm{g}$. If $\mathrm{g}$ is protected by a $\mathrm{RW}$ lock (either task-fair or phase-fair) and if $T_{i}^{j}$ 's requests interfere with at most $w$ write requests, then $b_{w} \leq 2 w+c_{\mathcal{W}}$.

Proof. By induction over $w$.

Base case $w=0$ : if there are no interfering write requests, then $T_{i}^{j}$,s read requests (if any) do not incur blocking at all (since interfering read requests do not block each other), and $T_{i}^{j}$,s write requests (if any) can only be blocked by one reader phase each. Hence, $T_{i}^{j}$ is blocked by at most $c_{\mathcal{W}}$ reader phases, and thus $b_{w} \leq 2 w+c_{\mathcal{W}}$. This is illustrated in inset (a) of Fig. 6 .

Induction step $w \rightarrow w+1$ : adding one interfering write request $\mathcal{W}^{x}$ increases the number of phases blocking $T_{i}^{j}$ by at most two: firstly, $\mathcal{W}^{x}$ can directly block $T_{i}^{j}$ once, and, secondly, $\mathcal{W}^{x}$ can itself be blocked by one additional reader phase $\mathcal{R}^{y}$, which then transitively also blocks $T_{i}^{j}$. This is illustrated in inset (b) of Fig. 6. Since by the induction hypothesis $b_{w} \leq 2 w+c_{\mathcal{W}}$ and $b_{w+1} \leq b_{w}+2$, it follows that $b_{w+1} \leq b_{w}+2 \leq\left(2 w+c_{\mathcal{W}}\right)+2=2(w+1)+c_{\mathcal{W}}$.

Note that Lemma 4 holds irrespective of the number of interfering read requests $r$. If $r \leq w$, then Lemma 4 trivially holds by Lemma 2 since, in this case, $2 w+c_{\mathcal{W}} \geq w+r$; if $r>w$, then some of the interfering read requests are satisfied concurrently, as read requests can only block each other when they are "separated" by a write request (of which there are, by assumption, only $w$ ).

Lemma 5. Let $c_{\mathcal{W}}$ be the number of outermost write requests that a job $T_{i}^{j}$ issues for resources in group g. If $T_{i}^{j}$ is blocked by $b$ interfering requests across all outermost requests issued for resources in group $g$, then $T_{i}^{j}$ is blocked by at least $\max \left(0,\left\lceil\frac{b-c_{\mathcal{W}}}{2}\right\rceil\right)$ interfering writer phases.

Proof. By contradiction. Assume $\frac{b-c_{\mathcal{W}}}{2}>0$ (otherwise the claim is trivially true), and further assume that $T_{i}^{j}$ interfered with $w<\frac{b-c_{\mathcal{W}}}{2}$ writer phases $\left(w \in \mathbb{N}_{0}\right.$; note the absence of the ceiling). Then, by Lemma 4 :

$$
\begin{aligned}
b & \leq 2 w+c_{\mathcal{W}} \\
\Rightarrow b & <2\left(\frac{b-c_{\mathcal{W}}}{2}\right)+c_{\mathcal{W}} \\
\Leftrightarrow b & <b .
\end{aligned}
$$

Contradiction. Hence, $\frac{b-c_{\mathcal{W}}}{2}$, which may not be integral, is a lower bound on $w$. Since $T_{i}^{j}$ can only be blocked by an integral number of writer phases, the claim follows.

\section{A.5 Task-Fair Mutex Locks}

With task-fair mutex locks (e.g., ticket locks [27]), both readers and writers gain exclusive access to resource groups. Hence, when bounding direct blocking under task-fair mutex locks, the request type (read or write) is irrelevant. 
Theorem 1. Let $c=x \operatorname{count}\left(T_{i}, g\right)$ be the maximum number of requests issued by any $T_{i}^{j}$ for resources in group $g$, and let $t=\mathrm{c}\left(T_{i}\right)$ be the maximum interval that any any $T_{i}^{j}$ can be active. Under global scheduling, direct blocking incurred by any $T_{i}^{j}$ through group $g$ is bounded by

$$
d b g_{G L O B A L}^{M T X}\left(T_{i}, g\right)=\operatorname{total}((m-1) \cdot c, \operatorname{xifs}(\text { others }(i), g, t, c)) .
$$

Proof. Under global scheduling, jobs of every other task in the system can cause interference while $T_{i}^{j}$ is active. By Lemma 1 , at most $(m-1)$ remote jobs can cause direct blocking (per request). Hence, at most $(m-1) \cdot c$ requests interfering requests can block $T_{i}^{j}$ in total. By Lemma 3, each remote job can block $T_{i}$ with no more than $c$ interfering requests. Therefore, the maximum blocking duration is bounded by the sum of the durations of the $(m-1) \cdot c$ longest requests in xifs $($ others $(i), g, t, c)$.

Theorem 2. Let $c=x \operatorname{xcount}\left(T_{i}, g\right)$ be the maximum number of requests issued by any $T_{i}^{j}$ for resources in group $g$, and let $t=\mathrm{c}\left(T_{i}\right)$ be the length of the maximum interval in which any $T_{i}^{j}$ can be active. Under partitioned scheduling, direct blocking incurred by any $T_{i}^{j}$ through group $g$ is bounded by

$$
\operatorname{dbg}_{\text {PART }}^{M T X}\left(T_{i}, g\right)=\operatorname{total}\left((m-1) \cdot c, p x i f s\left(T_{i}, g, t, c\right)\right) .
$$

Proof. Similarly to Theorem 1, the maximum number of blocking requests is bounded by $(m-1) \cdot c$. However, the set of potentially interfering tasks is limited to remote partitions. Therefore, the maximum blocking duration is bounded by the sum of the durations of the $(m-1) \cdot c$ longest requests in $\operatorname{pxifs}\left(T_{i}, g, t, c\right)$.

Note that Eq. (29)—but not Eq. (28)—was derived previously in [11].

\section{A.6 Task-Fair RW Locks}

With task-fair RW locks, writers gain exclusive access to resource groups, whereas reads may access resource groups concurrently. However, jobs gain access to resource groups in strict FIFO order: a writer that is directly blocked by a reader transitively blocks later-arriving readers while it is waiting.

\section{A.6.1 Bounding Direct Blocking under Global Scheduling}

In the following discussion, let $c_{\mathcal{R}}=\operatorname{rcount}\left(T_{i}, g\right), c_{\mathcal{W}}=w \operatorname{count}\left(T_{i}, g\right)$, and $c=c_{\mathcal{R}}+c_{\mathcal{W}}$ denote the maximum number of read, write, and all requests issued by any job $T_{i}^{j}$ of $T_{i}$ for resources in group $g$ (respectively), and let $t=\mathrm{c}\left(T_{i}\right)$ denote the length of the maximum interval that $T_{i}^{j}$ can be active. Further, let

$$
R=\operatorname{rifs}(\text { others }(i), g, t, c), W=\text { wifs }(\text { others }(i), g, t, c) \text {, and } X=x i f s(\text { others }(i), g, t, c)
$$

denote the worst-case read, write, and request interference (respectively) incurred by $T_{i}^{j}$ during any interval of length $t$. By Lemmas 1, 2, and 4, at most

$$
b=\min \left(|W|+|R|,|W| \cdot 2+c_{\mathcal{W}},(m-1) \cdot c\right)
$$

phases can block requests of $T_{i}^{j}$. Similarly to Theorem 1 , one upper bound for the maximum duration of blocking incurred is the sum of the $b$ longest interfering requests in $X$. However, if writer phases are short and there are many long reader phases, then this simple bound could be very pessimistic as it does not differentiate between reader and writer phases, i.e., the bound could equal the sum of the $b$ longest reader phases, even though that is clearly not a feasible scenario. By Lemma 5 , if $T_{i}^{j}$ is blocked by $b$ phases, then at least

$$
w=\max \left(0,\left\lceil\frac{b-c_{\mathcal{W}}}{2}\right\rceil\right)
$$

writer phases block $T_{i}^{j}$. This can be used to obtain a second, potentially tighter bound: the sum of the the durations of the $w$ longest requests in $W$ and the $b-w$ longest requests in $X$ (that are not also among the $w$ longest requests in $W$ ) also bounds $T_{i}^{j}$, s worst-case blocking time (the $b-w$ requests can be either read or write requests). However, note that the latter bound can be more pessimistic than the former bound in pathological cases wherein few remote tasks have both long read and long (but slightly shorter) writer requests because then the bound may represent a scenario in which some task(s) block $T_{i}^{j}$ with more than $c$ requests, which is impossible (by Lemma 3). Hence, it is beneficial to compute both bounds. 
Theorem 3. Under global scheduling, if $g$ is protected by a task-fair $R W$ lock, then blocking incurred by $T_{i}^{j}$ through group $g$ is bounded by

$$
d b g_{G L O B A L}^{T F}\left(T_{i}, g\right)=\min (\operatorname{total}(b, X), \operatorname{total}(w, W)+\operatorname{total}(b-w, X \backslash \text { longest }(w, W))) .
$$

Proof. Follows from the preceding discussion.

\section{A.6.2 Bounding Direct Blocking under Partitioned Scheduling}

The only difference of blocking under partitioned scheduling to blocking under global scheduling is how read, write, and request interference is computed as only tasks belonging to remote partitions must be considered. Hence, assume $t, c_{\mathcal{R}}, c_{\mathcal{W}}, c$, $b$, and $w$ are defined as before in Sec. A.6.1, and let

$$
R=\operatorname{prifs}\left(T_{i}, g, t, c\right), W=\operatorname{pwifs}\left(T_{i}, g, t, c\right), \text { and } X=\operatorname{pxifs}\left(T_{i}, g, t, c\right)
$$

denote the worst-case read, write, and request interference (respectively) incurred by $T_{i}^{j}$ during any interval of length $t$. Note that, by definition, $|X| \leq(m-1) \cdot c$ (see Eq. (20)).

Theorem 4. Under partitioned scheduling, if $g$ is protected by a task-fair $R W$ lock, then blocking incurred by $T_{i}^{j}$ through group $g$ is bounded by

$$
d b g_{\text {PART }}^{T F}\left(T_{i}, g\right)=\min (\operatorname{total}(b, X), \operatorname{total}(w, W)+\operatorname{total}(b-w, X \backslash \text { longest }(w, W))) .
$$

Proof. Similar to the proof of Theorem 3.

\section{A.7 Phase-Fair RW Locks}

With phase-fair RW locks, writers gain access in strict FIFO order with respect to other writers. Readers and writers, however, are only ordered with regard to phases. Under contention, reader and writer phases alternate, and with the beginning of a reader phase, all currently blocked readers gain concurrent access. Hence, a reader may gain access to a resource group prior to an earlier-arrived writer.

Lemma 6. If a resource group $g$ is protected by a phase-fair $R W$ lock, then a read request $\mathcal{R}$ for a resource in $g$ is blocked by at most one writer phase and one reader phase.

Proof. Follows from the definition of phase-fairness (see Sec. 3).

Lemma 7. If a resource group $g$ is protected by a phase-fair $R W$ lock, then a write request $\mathcal{W}$ for a resource in $g$ is blocked by at most $m-1$ writer phases and $m-1$ reader phases.

Proof. Since write requests are satisfied in FIFO order with respect to each other, at most $m-1$ writer phases can block $\mathcal{W}$ by Lemma 1. However, since writer and reader phases alternate and because later-arriving readers can initiate reader phases that block $\mathcal{W}$, in the worst-case, every of the $m$ writer phases (including $\mathcal{W}$ ) can be separated by a reader phase, for a total of $m-1$ reader phases.

Lemma 8. Let $c_{\mathcal{R}}$ be the number of outermost read requests and $c_{\mathcal{W}}$ the number of outermost write requests that a job $T_{i}^{j}$ issues for resources in group $g$ (which is protected by a phase-fair $R W$ lock). Jobs of a task $T_{x}$ can block $T_{i}^{j}$ with at most $c_{\mathcal{R}}+c_{\mathcal{W}}$ interfering outermost write requests and $c_{\mathcal{R}}+(m-1) \cdot c_{\mathcal{W}}$ outermost read requests.

Proof. By Lemmas 3 and 6, jobs of $T_{x}$ can block $T_{i}^{j}$ with a write request at most once each time $T_{i}^{j}$ issues a request (either read or write) for resources in $g$. Hence, $T_{x}$ blocks $T_{i}^{j}$ with at most $c_{\mathcal{R}}+c_{\mathcal{W}}$ write requests. By Lemma 6 , jobs of $T_{x}$ can block $T_{i}^{j}$ with a read request at most once each time that $T_{i}^{j}$ issues a read request for resources in $g$. By Lemma 7, no more than $m-1$ read requests issued by jobs of $T_{x}$ can block $T_{i}^{j}$ each time that $T_{i}^{j}$ issues a write request for resources in $g$. Hence, $T_{x}$ blocks $T_{i}^{j}$ with at most $c_{\mathcal{R}}+(m-1) \cdot c_{\mathcal{W}}$ read requests. 


\section{A.7.1 Bounding Direct Blocking under Global Scheduling}

As before, let $c_{\mathcal{R}}=\operatorname{rcount}\left(T_{i}, g\right), c_{\mathcal{W}}=w \operatorname{count}\left(T_{i}, g\right)$, and $c=c_{\mathcal{R}}+c_{\mathcal{W}}$ denote the maximum number of read, write, and all requests issued by any job $T_{i}^{j}$ of $T_{i}$ for resources in group $g$ (respectively), and let $t=\mathrm{c}\left(T_{i}\right)$ denote the length of the maximum interval that $T_{i}^{j}$ can be active. By Lemma 8, a remote task $T_{x}$ can block $T_{i}^{j}$ with at most $q_{w}=c_{\mathcal{R}}+c_{\mathcal{W}}$ write requests and at most $q_{r}=c_{\mathcal{R}}+c_{\mathcal{W}} \cdot(m-1)$ read requests. Therefore, let

$$
R=\operatorname{rifs}\left(\operatorname{others}(i), g, t, q_{r}\right), W=\text { wifs }\left(\operatorname{others}(i), g, t, q_{w}\right), \text { and } X=\operatorname{rifs}\left(\operatorname{others}(i), g, t, q_{r}\right)
$$

denote the worst-case read, write, and request interference (respectively) incurred by $T_{i}^{j}$ during any interval of length $t .^{7}$ By Lemmas 6 and 7, $T_{i}^{j}$ is blocked by no more than $b_{1}=c_{\mathcal{R}}+c_{\mathcal{W}} \cdot(m-1)$ reader and writer phases each. A (potentially pessimistic) bound on worst-case blocking is hence given by the sum of the durations of $b_{1}$ longest requests in $R$ and the $b_{1}$ longest requests in $W$.

Similarly to Theorem 3, a second bound can be obtained by considering the lower bound on the number of blocking writer phases (i.e., Lemma 5) . By Lemmas 2 and 4, at most

$$
b_{2}=\min \left(|W|+|R|,|W| \cdot 2+c_{\mathcal{W}}, 2 \cdot b_{1}\right)
$$

phases (either read or write) can block requests of $T_{i}^{j}$. By Lemma 5 , if $T_{i}^{j}$ is blocked by $b_{2}$ phases, then at least

$$
w=\max \left(0,\left\lceil\frac{b_{2}-c_{\mathcal{W}}}{2}\right\rceil\right)
$$

writer phases block $T_{i}^{j}$. This yields a second bound: the sum of the the durations of the $w$ longest requests in $W$ and the $b_{2}-w$ longest requests in $X$ (that are not among the $w$ longest requests in $W$ ) also bounds $T_{i}^{j}$,s worst-case blocking time.

Theorem 5. Under global scheduling, if $g$ is protected by a phase-fair $R W$ lock, then blocking incurred by $T_{i}^{j}$ through group $g$ is bounded by

$$
d b g_{G L O B A L}^{P F}\left(T_{i}, g\right)=\min \left(\operatorname{total}\left(b_{1}, W\right)+\operatorname{total}\left(b_{1}, R\right), \operatorname{total}(w, W)+\operatorname{total}\left(b_{2}-w, X \backslash \text { longest }(w, W)\right)\right) .
$$

Proof. Follows from the preceding discussion.

\section{A.7.2 Bounding Direct Blocking under Partitioned Scheduling}

As before with Theorem 4, a bound for direct blocking with phase-fair RW locks under partitioned scheduling can be obtained analogously to previous derivation - the bounds only differ in the computation of read and write interference. Let

$$
R=\operatorname{prifs}\left(T_{i}, g, t, q_{r}\right), W=\operatorname{pwifs}\left(T_{i}, g, t, q_{w}\right) \text { and } X=\operatorname{pxifs}\left(T_{i}, g, t, q_{r}\right)
$$

denote the worst-case write and read interference (respectively) incurred by $T_{i}^{j}$.

Theorem 6. Under partitioned scheduling, if $g$ is protected by a phase-fair $R W$ lock, then blocking incurred by $T_{i}^{j}$ through group $g$ is bounded by

$$
d b g_{P A R T}^{P F}\left(T_{i}, g\right)=\min \left(\operatorname{total}\left(b_{1}, W\right)+\operatorname{total}\left(b_{1}, R\right), \operatorname{total}(w, W)+\operatorname{total}\left(b_{2}-w, X \backslash \text { longest }(w, W)\right)\right) .
$$

Proof. Similar to Theorem 6.

\footnotetext{
${ }^{7}$ Since tasks are sequential, a remote task $T_{x}$ cannot execute an outermost read request and an outermost write request concurrently, and hence can block $T_{i}^{j}$ with at $\operatorname{most} \max \left(q_{r}, q_{w}\right)=q_{r}$ requests of any kind.
} 


\section{A.8 Arrival Blocking}

Arrival blocking is bounded by the length of the longest non-preemptive section. Since requests are executed non-preemptively, the bound on the length of a non-preemptive section due to a request $\mathcal{X}$ depends on both the bound on per-request worst-case blocking time, denoted $b(\mathcal{X})$, and the maximum request duration $|\mathcal{X}|$. A bound on worst-case arrival blocking incurred by jobs of task $T_{i}$ is hence given by

$$
\operatorname{AB}_{\text {GLOBAL }}\left(T_{i}\right)=\max \left(\left\{|\mathcal{X}|+b(\mathcal{X}) \mid \mathcal{X} \in \bigcup_{T_{x} \in \text { others }(i)}\left(\operatorname{reads}\left(T_{x}\right) \cup \operatorname{writes}\left(T_{x}\right)\right)\right\}\right)
$$

in the case of global scheduling and

$$
\operatorname{AB}_{\text {PART }}\left(T_{i}\right)=\max \left(\left\{|\mathcal{X}|+b(\mathcal{X}) \mid \mathcal{X} \in \bigcup_{T_{x} \in \operatorname{partition}\left(\mathrm{P}\left(T_{i}\right)\right)}\left(\operatorname{reads}\left(T_{x}\right) \cup \operatorname{writes}\left(T_{x}\right)\right)\right\}\right)
$$

in the case of partitioned scheduling. For each request $\mathcal{X}$, the per-request bound $b(\mathcal{X})$ can be obtained by computing $d b g\left(T_{x}, \operatorname{grp}(\operatorname{res}(\mathcal{X}))\right)$ while assuming that $\mathcal{X}$ is the only request issued by $T_{x}$ (as given by Theorems $1-6$, respectively), i.e., the worst-case blocking that $\mathcal{X}$ can incur is clearly no more than the worst-case blocking that $T_{x}$ would incur if $\mathcal{X}$ were the only request issued by $T_{x}$. Hence, $b(\mathcal{X})=d b g\left(T_{x}, \operatorname{grp}(\operatorname{res}(\mathcal{X}))\right)$ if $d b g\left(T_{x}, \operatorname{grp}(\operatorname{res}(\mathcal{X}))\right)$ is evaluated under the assumption that reads $\left(T_{x}\right) \cup$ writes $\left(T_{x}\right)=\{\mathcal{X}\}$.

\section{B A Phase-Fair Lock for Memory-Constrained Systems}

A phase-fair reader-writer lock implementation that only requires four bytes (i.e., one 32 bit word, as shown in Fig. 7) of memory is given in Listing 2. It closely resembles the implementation given in Listing 1 , hence we focus on the notable differences in the following discussion.

The four ticket counters, named rin, rout, win, and wout in Listing 1, are collapsed into four 7-bit-wide fields of the lock, as shown in Fig. 7. The bit fields are separated by one bit each that serves as an overflow guard. The ticket counters are updated with fetch-and-add, atomic-add, and atomic-sub operations in which the second operand has been shifted by the offset of the to-be-updated ticket counter. The respective offsets are given in lines 3-6 of Listing 2.

The position of the PHID bit and the PRES bit are reversed in order to overlay PHID with the least-significan bit of wout: bit zero is used to signal the presence of a writer $(P R E S)$, and bit one, which is also the least-significant bit of wout, is used by readers to tell consecutive writer phases apart $(P H I D)$.

Overflowing rin and win is avoided by clearing the respective overflow guard bit whenever it becomes set (lines 13-15 and 28-30). Overflowing rout is harmless since the state of the lock is truncated automatically processor when rout overflows. Allocating rout in the most-significant bits of the lock word is hence an optimization of the reader exit procedure. Since wout is never updated concurrently, a possible overflow can be detected before it is updated (lines 38-44). The overflow guard between win and wout is therefore unused, but cannot be allocated to either win or wout since they must be equal in size.

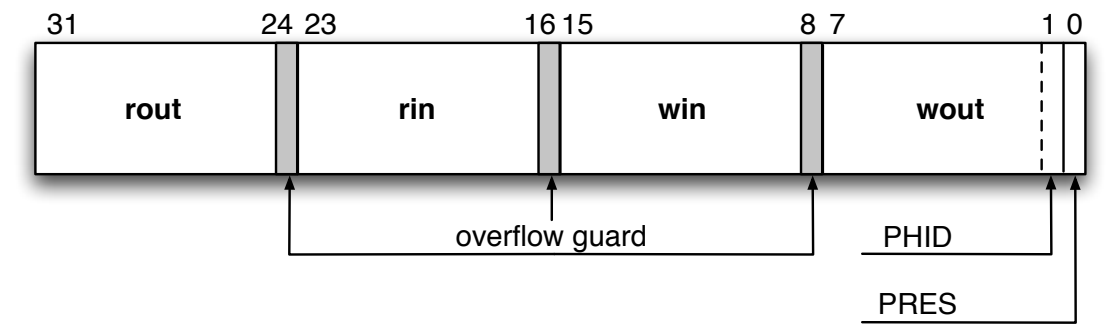

Figure 7: Bit fields in the 32-bit implementation of the phase-fair reader-writer lock. The four ticket counters rin, rout, win, and wout consist of seven bits each. Due to the limited size of the ticket counters, at most 127 readers and 127 writers may issue requests concurrently. Note that PHID is the least-significant bit of wout. 


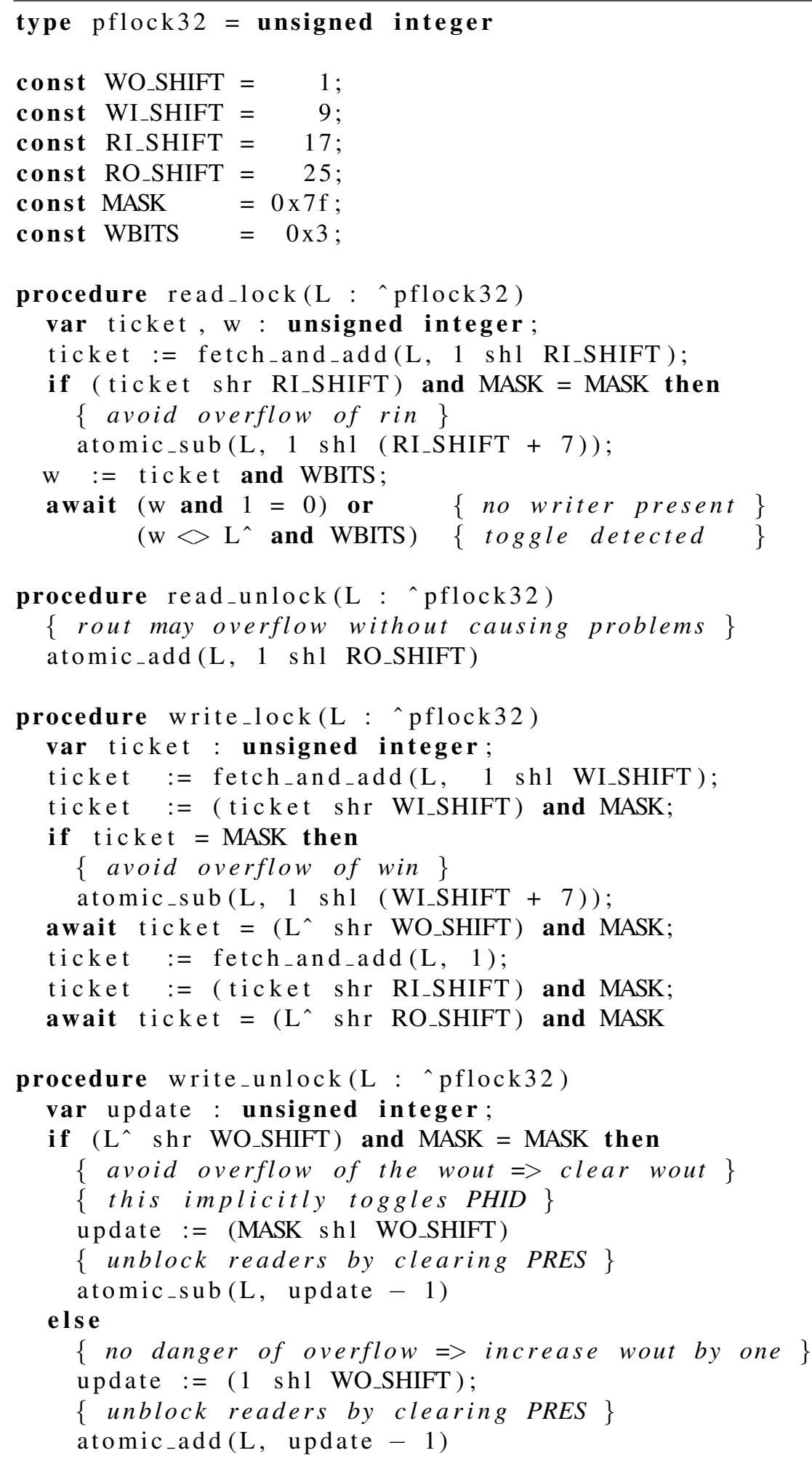

Listing 2: Implementation of a phase-fair reader-writer lock that fits within four bytes. 